Research Article

\title{
New Method of Solving the Economic Complex Systems
}

\author{
Ya-Juan Yang $\mathbb{D}^{1,}{ }^{1,2}$ Chung-Cheng Chen $\mathbb{D D}^{2},{ }^{2}$ and Yen-Ting Chen $\mathbb{D}^{3}$ \\ ${ }^{1}$ School of Business, Macau University of Science and Technology, Taipa, Macau \\ ${ }^{2}$ City College of Dongguan University of Technology, Dongguan, Guangdong, China \\ ${ }^{3}$ Department of Electrical Engineering, Graduate School, National Chung Hsing University, 145 Xingda Road, South District, \\ Taichung 402, Taiwan \\ Correspondence should be addressed to Chung-Cheng Chen; ccc49827@ms25.hinet.net
}

Received 18 September 2020; Revised 31 October 2020; Accepted 12 November 2020; Published 29 November 2020

Academic Editor: Lijun Pei

Copyright ( 2020 Ya-Juan Yang et al. This is an open access article distributed under the Creative Commons Attribution License, which permits unrestricted use, distribution, and reproduction in any medium, provided the original work is properly cited.

In this study, the authors first develop a direct method used to solve the linear nonhomogeneous time-invariant difference equation with the same number for inputs and outputs. Economic cybernetics is the crystallization for the integration of economics and cybernetics. It analyzes the stability, controllability, and observability of the economic system by establishing a system model and enables people to better understand the characteristics of the economic system and solve economic optimization problems. The economic model generally applies the discrete recurrence difference equation. The significant analytic approach for the difference equation is the $z$-transformation technique. The $z$-transformation state of the economic cybernetics state-space difference equation generally is a rational function with the same power for the numerator and the denominator. The proposed approach will take the place of the traditional methods without all annoying procedures involving the long division of some complicated polynomials, the expanded multiplication of many polynomial factors, the differentiation of some complicated polynomials, and the complex derivations of all partial fraction parameters. To highlight the novelty of this research, this study especially applies the proposed theorems originally belonging to engineering to the field of economic applications.

\section{Introduction}

Linear nonhomogeneous time-invariant difference equations are ubiquitous in many engineering and mathematical fields [1]. For example, they appear in the engineering theory of discrete-time systems and control theory of discrete-time systems as fundamental models of the discrete-time systems [2-4], and discrete-time signal processing as fundamental recurrence equations of sampled signals [5]. In algebraic combinatorics, they are also one of the most significant topics, tying different special sequences with their generating functions $[6,7]$.

It is well known that the system theory, the cybernetics, and the information theory are called the three theories of science today and have become the basic theories of the development for natural sciences and social sciences. According to the cybernetic view, the economic system is an organized and controlled system, and a series of economic activities such as production, consumption, distribution, and exchange are closely related. According to the scope of economic activities and the difference in economic structure, it can be divided into two categories: macroeconomic system and microeconomic system. Economic systems are often large systems that are complex. To achieve significant goals of optimal development, balance between supply and demand, optimal allocation of resources, and sustainable economic development, accurate quantitative analysis of the operation for the economic system must be conducted under the guidance of economic theory. Adjustment, control, decision-making, and planning are carried out, on the basis of which the total amount and structure of economic development are more reasonable. Obviously, economic cybernetics provides basic theory and more advanced methods for us to study complex economic systems.

The applications of economic cybernetics in the operation management and control of economic systems are 
mainly manifested in the following three aspects [8]:

(1) Economic planning. In the macroeconomic system, to ensure the stability, optimal and sustainable development of the national economic system, we not only need timely regulation of the fluctuations in the economic operation, but also make scientific medium-term and long-term planning for the operation of the economic system. Economic cybernetics is a powerful tool for formulating long-term development plans for economic development strategies, economic restructuring, economic operations, and economic management system reforms. In terms of microeconomic operation, it is necessary to improve the management level of enterprises, to improve the efficiency for input and output of enterprises, to make the development of enterprises conform to the direction of national industrial development, and to make the development of enterprises into the international track. Economic cybernetics is also an important tool for scientific development planning.

(2) Structural reform of the economic system. For the macroeconomic system and the microeconomic system, the operational structure of the system needs to be evaluated from the total amount and structure. In a sense, the structures of the system are more important, such as the first industrial structure, industry structure within an industry, product structure within the industry, savings and consumption structure, resource supply structure, investment structure, and consumption structure. In this regard, we can use economic cybernetics to establish a system model that analyzes the internal state variables of the system, so as to effectively avoid the obstacles for the economic system operation caused by the irrational structure, and provide the improvement of the economic system structure.

(3) Optimization of the operation of the economic system. The ideal operational goal of the economic system is to steadily increase the total amount of the various proportional structures within the system. Economic cybernetics analyzes the stability, controllability, and observability of the model by establishing a system model. It enables people to better understand the characteristics of the economic system and solve economic optimization problems.

Economic cybernetics is the science that applies cybernetics to study the laws of social economic system activities. It provides an efficient method for studying the function and efficiency of the socioeconomic system and plays an important role in improving the decision-making level of the socioeconomic system and promoting economic and social development. Economic cybernetics in the macroeconomic aspects of population control, ecological balance, resource optimization allocation, analysis of economic fluctuation cycle, fiscal and monetary policy design, equilibrium price calculation, and forecasting and in the microeconomic aspects of optimization for production resource allocation, enterprise structure reform, enterprise economic benefit analysis, supply and demand price analysis, and forecasting has good application prospects. Generally speaking, whether it is a macroeconomic system or a microeconomic system, the variables in the system are counted according to the discrete-time such as year, season, month, and day, which is consistent with the actual situation. Therefore, the economic cybernetics model generally adopts the discrete-time form, and its mathematical form of the state-space equation is the first-order difference equation $x(k+1)=$ $A x(k)+B x(k)$. System mathematics model is a system of difference equations in addition to economic cybernetics model $[9,10]$, especially applied to short- and long-term repeated game behaviors of two parallel supply chains based on government subsidy in the vehicle market [11], supply chain coordination in the presence of uncertain yield and demand [12], and a timebased pricing game in a competitive vehicle market regarding the intervention of carbon emission reduction [13], as well as HIV biomedical systems [14] and discrete-time systems [15]. The main mathematical tool for solving the difference equation is the $\mathrm{z}$-transformation in the signal system theory [16].

In this study, we develop a direct method used to solve the linear nonhomogeneous time-invariant difference equation with the same number for inputs and outputs. The $z$-transformation state of the economic cybernetics statespace difference equation generally is a rational function with the same power for the numerator and the denominator, and then the boresome processes involving the long division of both complicated polynomials, the expanded multiplication of all polynomial factors, the differentiation of both complicated polynomials, and the complex derivations of all partial fraction parameters are inevitable for existing traditional methods. For the rational function with the same power of the numerator and the denominator, the main traditional methods that exist are the following: (1) long-division and partial fraction expansion method [17]; (2) divided by $z$ and partial fraction expansion method [18-24]; (3) direct long-division method [20, 21]; (4) inversion integral method [21, 25-27]. The disadvantages of the long division and partial fraction expansion method involve three troublesome operations: the multiplication of all polynomial factors, the long division of both complicated polynomials $[20,21]$, and the derivations of the complicated partial fraction expansion variables. According to the $z$ transformed table, it is obvious to see the fact that almost all $z$-transformation formulas have a factor $z$ in the numerator. To consist with the Laplace transform for continuous-time system via partial fraction expansion technique, the divided by $z$ and partial fraction expansion method can be applied, and the function $F(z)$ will be rewritten as $F(z) / z$. However, this special $F(z)$ with $z$-factor will increase the burden for involving two troublesome operations: the multiplication of all polynomial factors and the differentiation of two complicated polynomials [18-24]. The direct long-division method only yields some finite terms of $F(k)$ and also needs two troublesome operations: the multiplication of all polynomial factors and the long division of two polynomials $[20,21]$. The disadvantages of the inversion integral method involve two tedious operations: the multiplication of all polynomial factors and the differentiation of both complex 
polynomials [21, 25-27]. To solve these difficulties of the existing traditional approaches, we have developed more direct theorems with shorter solving time than the traditional methods. Finally, to show the practical value of this study, we use the proposed direct method to solve the famous economic cybernetics in population prediction, analysis of market price balance, and loan economic activities. Major contributions of this study are summarized as follows:

(1) This study first develops a direct method used to solve the linear nonhomogeneous time-invariant difference equation with the same number for inputs and outputs.

(2) In this study, the proposed approach takes the place of the traditional methods without all annoying procedures involving (i) the long division of some complicated polynomials; (ii) the expanded multiplication of many polynomial factors; (iii) the differentiation of some complicated polynomials; (iv) the complex derivations of all partial fraction parameters.

(3) To highlight the novelty of this research, this study especially applies the proposed theorems originally belonging to engineering to the field of economic applications.

\section{Main Results}

Some simple direct theorems will be shown here to solve the inverse $z$-transformation for a rational function with the same power of the numerator and the denominator.

Theorem 1. Given a rational function $F(z)$ with distinct real poles, where the numerator and the denominator are in the same power

$$
\begin{aligned}
F(z) & =\frac{a_{n} z^{n}+a_{n-1} z^{n-1}+\cdots+a_{1} z^{1}+a_{0}}{b_{n} z^{n}+b_{n-1} z^{n-1}+\cdots+b_{1} z^{1}+b_{0}}=\frac{\left(a_{n} / b_{n}\right) z^{n}+\left(a_{n-1} / b_{n}\right) z^{n-1}+\cdots+\left(a_{1} / b_{n}\right) z^{1}+\left(a_{0} / b_{n}\right)}{z^{n}+\left(b_{n-1} / b_{n}\right) z^{n-1}+\cdots+\left(b_{1} / b_{n}\right) z^{1}+\left(b_{0} / b_{n}\right)} \\
& \equiv \frac{\left(a_{n} / b_{n}\right) z^{n}+\left(a_{n-1} / b_{n}\right) z^{n-1}+\cdots+\left(a_{1} / b_{n}\right) z^{1}+\left(a_{0} / b_{n}\right)}{\left(z-p_{1}\right)\left(z-p_{2}\right) \cdots\left(z-p_{n}\right)} \equiv \frac{N(z)}{\left(z-p_{1}\right)\left(z-p_{2}\right) \cdots\left(z-p_{n}\right)} \\
& =\left(\frac{a_{n}}{b_{n}}\right)+\frac{k_{1}}{z-p_{1}}+\frac{k_{2}}{z-p_{2}}+\cdots+\frac{k_{n}}{z-p_{n}}, \\
N(z) & \equiv\left(\frac{a_{n}}{b_{n}}\right) z^{n}+\left(\frac{a_{n-1}}{b_{n}}\right) z^{n-1}+\cdots+\left(\frac{a_{1}}{b_{n}}\right) z^{1}+\left(\frac{a_{0}}{b_{n}}\right), \\
k_{1} & =\left.\frac{N(z)}{\left(z-p_{1}\right)\left(z-p_{2}\right), \ldots,\left(z-p_{n}\right)}\left(z-p_{1}\right)\right|_{z=p_{1}}, \\
k_{2} & =\left.\frac{N(z)}{\left(z-p_{1}\right)\left(z-p_{2}\right), \ldots,\left(z-p_{n}\right)}\left(z-p_{2}\right)\right|_{z=p_{2}}, \\
k_{n} & =\left.\frac{N(z)}{\left(z-p_{1}\right)\left(z-p_{2}\right), \ldots,\left(z-p_{n}\right)}\left(z-p_{n}\right)\right|_{z=p_{n} .}
\end{aligned}
$$

After long division, $F(z)$ can be written to be

$$
\begin{aligned}
F(z) & =\left(\frac{a_{n}}{b_{n}}\right)+\frac{a_{n-1}{ }^{n} z^{n-1}+a_{n-2}{ }^{\prime} z^{n-2}+\cdots+a_{1}^{\prime} z^{1}+a_{0}^{\prime}}{\left(z-p_{1}\right)\left(z-p_{2}\right), \ldots,\left(z-p_{n}\right)} \\
& \equiv\left(\frac{a_{n}}{b_{n}}\right)+\left[\frac{N^{\prime}(z)}{\left(z-p_{1}\right)\left(z-p_{2}\right), \ldots,\left(z-p_{n}\right)}\right] \\
& =\left(\frac{a_{n}}{b_{n}}\right)+\left[\frac{k_{1}^{\prime}}{z-p_{1}}+\frac{k_{2}^{\prime}}{z-p_{2}}+\cdots+\frac{k_{n}^{\prime}}{z-p_{n}}\right], \\
N^{\prime}(z) & \equiv a_{n-1}^{\prime} z^{n-1}+a_{n-2}^{\prime} z^{n-2}+\cdots+a_{1}^{\prime} z^{1}+a_{0}^{\prime},
\end{aligned}
$$

where

$$
\begin{aligned}
& k_{1}^{\prime}=\left.\frac{N^{\prime}(z)}{\left(z-p_{1}\right)\left(z-p_{2}\right), \ldots,\left(z-p_{n}\right)}\left(z-p_{1}\right)\right|_{z=p_{1}}, \\
& k_{2}^{\prime}=\left.\frac{N^{\prime}(z)}{\left(z-p_{1}\right)\left(z-p_{2}\right), \ldots,\left(z-p_{n}\right)}\left(z-p_{2}\right)\right|_{z=p_{2}}, \\
& k_{n}^{\prime}=\left.\frac{N^{\prime}(z)}{\left(z-p_{1}\right)\left(z-p_{2}\right), \ldots,\left(z-p_{n}\right)}\left(z-p_{n}\right)\right|_{z=p_{n}} .
\end{aligned}
$$

Then, we can get

$$
k_{1}=k_{1}^{\prime}, k_{2}=k_{2}^{\prime}, \ldots, k_{n}=k_{n}^{\prime},
$$


that is, the long division operation truly is not necessary. The inverse transformation of $F(z)$ is

$$
\begin{aligned}
f(k) & =\left(\frac{a_{n}}{b_{n}}\right) \delta(k)+k_{1}\left(p_{1}\right)^{k-1} u_{s}(k-1)+k_{2}\left(p_{2}\right)^{k-1} u_{s}(k-1)+\cdots+k_{n}\left(p_{n}\right)^{k-1} u_{s}(k-1) \\
& =\left(\frac{a_{n}}{b_{n}}\right) \delta(k)+\left.k_{1}\left(p_{1}\right)^{k} u_{s}(k)\right|_{k=k-1}+\left.k_{2}\left(p_{2}\right)^{k} u_{s}(k)\right|_{k=k-1}+\cdots+\left.k_{n}\left(p_{n}\right)^{k} u_{s}(k)\right|_{k=k-1} .
\end{aligned}
$$

Proof. From (1) and (6), we get

Then,

$$
N(z)=\left(\frac{a_{n}}{b_{n}}\right)\left(z-p_{1}\right)\left(z-p_{2}\right), \ldots,\left(z-p_{n}\right)+N^{\prime}(z) .
$$

$$
\begin{aligned}
& k_{1}^{\prime}=\left.\frac{N^{\prime}(z)}{\left(z-p_{1}\right)\left(z-p_{2}\right), \ldots,\left(z-p_{n}\right)}\left(z-p_{1}\right)\right|_{z=p_{1}} \\
& =\left.\frac{\left(a_{n} / b_{n}\right)\left(z-p_{1}\right)\left(z-p_{2}\right), \ldots,\left(z-p_{n}\right)+N^{\prime}(z)}{\left(z-p_{1}\right)\left(z-p_{2}\right), \ldots,\left(z-p_{n}\right)}\left(z-p_{1}\right)\right|_{z=p_{1}} \\
& =\left.\frac{N(z)}{\left(z-p_{1}\right)\left(z-p_{2}\right), \ldots,\left(z-p_{n}\right)}\left(z-p_{1}\right)\right|_{z=p_{1}}=k_{1} \text {, } \\
& k_{2}^{\prime}=\left.\frac{N^{\prime}(z)}{\left(z-p_{1}\right)\left(z-p_{2}\right), \ldots,\left(z-p_{n}\right)}\left(z-p_{2}\right)\right|_{z=p_{2}} \\
& =\left.\frac{\left(a_{n} / b_{n}\right)\left(z-p_{1}\right)\left(z-p_{2}\right), \ldots,\left(z-p_{n}\right)+N^{\prime}(z)}{\left(z-p_{1}\right)\left(z-p_{2}\right), \ldots,\left(z-p_{n}\right)}\left(z-p_{2}\right)\right|_{z=p_{2}} \\
& =\left.\frac{N(z)}{\left(z-p_{1}\right)\left(z-p_{2}\right), \ldots,\left(z-p_{n}\right)}\left(z-p_{2}\right)\right|_{z=p_{2}}=k_{2} \text {, } \\
& k_{n}^{\prime}=\left.\frac{N^{\prime}(z)}{\left(z-p_{1}\right)\left(z-p_{2}\right), \ldots,\left(z-p_{n}\right)}\left(z-p_{n}\right)\right|_{z=p_{n}} \\
& =\left.\frac{\left(a_{n} / b_{n}\right)\left(z-p_{1}\right)\left(z-p_{2}\right), \ldots,\left(z-p_{n}\right)+N^{\prime}(z)}{\left(z-p_{1}\right)\left(z-p_{2}\right), \ldots,\left(z-p_{n}\right)}\left(z-p_{n}\right)\right|_{z=p_{n}} \\
& =\left.\frac{N(z)}{\left(z-p_{1}\right)\left(z-p_{2}\right), \ldots,\left(z-p_{n}\right)}\left(z-p_{n}\right)\right|_{z=p_{n}}=k_{n} .
\end{aligned}
$$

Define a rational function with single pole,

$$
G(z)=\frac{1}{z-p} \text {. }
$$

Utilizing the inverse $z$-transformation theorem of Cauchy residue calculus yields

$$
g(k)=\text { Residues }\left[\frac{1}{z-p}\left(z^{k-1}\right)\right] \text {, }
$$

where Residues $\left[1 / z-p\left(z^{k-1}\right)\right]$ denote the sum of residue values for the function $1 / z-p\left(z^{k-1}\right)$. For nonpositive values $k$, 


$$
\begin{aligned}
g(k)= & \text { Residues }\left[\frac{1}{(z-p) z^{1-k}}\right] \\
= & \text { Residue } \frac{1}{(z-p) z^{1-k}}+\underset{z=0}{\operatorname{Residue}} \frac{1}{(z-p) z^{1-k}} \\
= & \left.\frac{1}{(-k) !} \frac{\mathrm{d}^{(-k)}}{\mathrm{d} z^{(-k)}} \frac{1}{z-p}\right|_{z=0}+\left.\frac{1}{(0) !} \frac{\mathrm{d}^{(0)}}{\mathrm{d} z^{(0)}} \frac{1}{(z)^{1-k}}\right|_{z=p} \\
= & -\frac{1}{(-k) !}((-k)(-k-1), \ldots,(2)(1)) \frac{1}{(p)^{1-k}} \\
& +\frac{1}{(p)^{1-k}}=0
\end{aligned}
$$

Therefore, from equations (1) and (6), we get the inverse $z$-transformation of $F(z)$ as

$$
\begin{aligned}
f(k)= & \left(\frac{a_{n}}{b_{n}}\right) \delta(k)+k_{1}\left(p_{1}\right)^{k-1} u_{s}(k-1) \\
& +k_{2}\left(p_{2}\right)^{k-1} u_{s}(k-1)+\cdots+k_{n}\left(p_{n}\right)^{k-1} u_{s}(k-1) .
\end{aligned}
$$

Theorem 2. Knowing a rational function $F(z)$ with multiple poles, where the numerator and the denominator are in the same power,

For positive values $k$,

$$
\begin{aligned}
g(k) & =\text { Residues }\left[\frac{1}{(z-p)}\left(z^{k-1}\right)\right]=\underset{z=p}{\operatorname{Residue}} \frac{1}{(z-p)}\left(z^{k-1}\right) \\
& =\left.\frac{1}{(0) !} \frac{\mathrm{d}^{(0)}}{\mathrm{d} z^{(0)}} \frac{1}{(z-p)}\left(z^{k-1}\right)(z-p)\right|_{z=p}=(p)^{k-1} .
\end{aligned}
$$

$$
\begin{aligned}
& F(z)=\frac{a_{n} z^{n}+a_{n-1} z^{n-1}+\cdots+a_{1} z^{1}+a_{0}}{b_{n} z^{n}+b_{n-1} z^{n-1}+\cdots+b_{1} z^{1}+b_{0}}=\frac{\left(a_{n} / b_{n}\right) z^{n}+\left(a_{n-1} / b_{n}\right) z^{n-1}+\cdots+\left(a_{1} / b_{n}\right) z^{1}+\left(a_{0} b_{n}\right)}{z^{n}+\left(b_{n-1} / b_{n}\right) z^{n-1}+\cdots+\left(b_{1} / b_{n}\right) z^{1}+\left(b_{0} / b_{n}\right)} \\
& \equiv \frac{\left(a_{n} / b_{n}\right) z^{n}+\left(a_{n-1} / b_{n}\right) z^{n-1}+\cdots+\left(a_{1} / b_{n}\right) z^{1}+\left(a_{0} / b_{n}\right)}{\left(z-p_{1}\right)\left(z-p_{2}\right) \cdots\left(z-p_{n-r}\right)\left(z-p_{r}\right)^{r}} \equiv \frac{N(z)}{\left(z-p_{1}\right)\left(z-p_{2}\right) \cdots\left(z-p_{n-r}\right)\left(z-p_{r}\right)^{r}} \\
& =\left(\frac{a_{n}}{b_{n}}\right)+\left[\frac{k_{1}}{z-p_{1}}+\frac{k_{2}}{z-p_{2}}+\cdots+\frac{k_{n-r}}{z-p_{n-r}}+\left(\frac{A_{1}}{z-p_{r}}+\frac{A_{2}}{\left(z-p_{r}\right)^{2}}+\cdots+\frac{A_{r}}{\left(z-p_{r}\right)^{r}}\right)\right] \\
& k_{1}=\left.\frac{N(z)}{\left(z-p_{1}\right)\left(z-p_{2}\right), \ldots,\left(z-p_{n-r}\right)\left(z-p_{r}\right)^{r}}\left(z-p_{1}\right)\right|_{z=p_{1}} \\
& k_{2}=\left.\frac{N(z)}{\left(z-p_{1}\right)\left(z-p_{2}\right), \ldots,\left(z-p_{n-r}\right)\left(z-p_{r}\right)^{r}}\left(z-p_{2}\right)\right|_{z=p_{2}}, \\
& k_{n-r}=\left.\frac{N(z)}{\left(z-p_{1}\right)\left(z-p_{2}\right), \ldots,\left(z-p_{n-r}\right)\left(z-p_{r}\right)^{r}}\left(z-p_{n-r}\right)\right|_{z=p_{n-r}}, \\
& A_{r}=\left.\frac{1}{0 !} \frac{d^{0}}{d z^{0}} \frac{N(z)}{\left(z-p_{1}\right)\left(z-p_{2}\right), \ldots,\left(z-p_{n-r}\right)\left(z-p_{r}\right)^{r}}\left(z-p_{r}\right)^{r}\right|_{z=p_{r}}, \\
& A_{r-1}=\left.\frac{1}{1 !} \frac{d^{1}}{d z^{1}} \frac{N(z)}{\left(z-p_{1}\right)\left(z-p_{2}\right), \ldots,\left(z-p_{n-r}\right)\left(z-p_{r}\right)^{r}}\left(z-p_{r}\right)^{r}\right|_{z=p_{1}}, \\
& A_{1}=\left.\frac{1}{(r-1) !} \frac{d^{(r-1)}}{d z^{r-1}} \frac{N(z)}{\left(z-p_{1}\right)\left(z-p_{2}\right), \ldots,\left(z-p_{n-r}\right)\left(z-p_{r}\right)^{r}}\left(z-p_{r}\right)^{r}\right|_{z=p_{1}}, \\
& N(z) \equiv\left(\frac{a_{n}}{b_{n}}\right) z^{n}+\left(\frac{a_{n-1}}{b_{n}}\right) z^{n-1}+\cdots+\left(\frac{a_{1}}{b_{n}}\right) z^{1}+\left(\frac{a_{0}}{b_{n}}\right) \text {. }
\end{aligned}
$$


After a long division, $F(z)$ can be assumed to be

$$
\begin{aligned}
F(z) & =\left(\frac{a_{n}}{b_{n}}\right)+\frac{a_{n-1}{ }^{\prime} z^{n-1}+a_{n-2}{ }^{n-2}+\cdots+a_{1}^{\prime} z^{1}+a_{0}^{\prime}}{\left(z-p_{1}\right)\left(z-p_{2}\right), \ldots,\left(z-p_{n-r}\right)\left(z-p_{r}\right)^{r}} \\
& \equiv\left(\frac{a_{n}}{b_{n}}\right)+\left[\frac{N^{\prime}(z)}{\left(z-p_{1}\right)\left(z-p_{2}\right), \ldots,\left(z-p_{n-r}\right)\left(z-p_{r}\right)^{r}}\right] \\
& =\left(\frac{a_{n}}{b_{n}}\right)+\left[\frac{k_{1}^{\prime}}{z-p_{1}}+\frac{k_{2}^{\prime}}{z-p_{2}}+\cdots+\frac{k_{n-r}^{\prime}}{z-p_{n-r}}+\left(\frac{A_{1}^{\prime}}{z-p_{r}}+\frac{A_{2}^{\prime}}{\left(z-p_{r}\right)^{2}}+\cdots+\frac{A_{r}^{\prime}}{\left(z-p_{r}\right)^{r}}\right)\right], \\
N^{\prime}(z) & \equiv a_{n-1}{ }^{\prime} z^{n-1}+a_{n-2} z^{n-2}+\cdots+a_{1}^{\prime} z^{1}+a_{0}^{\prime},
\end{aligned}
$$

where

$$
\begin{aligned}
k_{1}^{\prime} & =\left.\frac{N^{\prime}(z)}{\left(z-p_{1}\right)\left(z-p_{2}\right), \ldots,\left(z-p_{n-r}\right)\left(z-p_{r}\right)^{r}}\left(z-p_{1}\right)\right|_{z=p_{1}}, \\
k_{2}^{\prime} & =\left.\frac{N^{\prime}(z)}{\left(z-p_{1}\right)\left(z-p_{2}\right), \ldots,\left(z-p_{n-r}\right)\left(z-p_{r}\right)^{r}}\left(z-p_{2}\right)\right|_{z=p_{2}}, \\
k_{n-r}^{\prime} & =\left.\frac{N^{\prime}(z)}{\left(z-p_{1}\right)\left(z-p_{2}\right), \ldots,\left(z-p_{n-r}\right)\left(z-p_{r}\right)^{r}}\left(z-p_{n-r}\right)\right|_{z=p_{n-r}}, \\
A_{r}^{\prime} & =\left.\frac{1}{0 !} \frac{d^{0}}{d z^{0}} \frac{N^{\prime}(z)}{\left(z-p_{1}\right)\left(z-p_{2}\right), \ldots,\left(z-p_{n-r}\right)\left(z-p_{r}\right)^{r}}\left(z-p_{r}\right)^{r}\right|_{z=p_{r}}, \\
A_{r-1}^{\prime} & =\left.\frac{1}{1 !} \frac{d^{1}}{d z^{1}} \frac{N^{\prime}(z)}{\left(z-p_{1}\right)\left(z-p_{2}\right), \ldots,\left(z-p_{n-r}\right)\left(z-p_{r}\right)^{r}}\left(z-p_{r}\right)^{r}\right|_{z=p_{1}}, \\
A_{1}^{\prime} & =\left.\frac{1}{(r-1) !} \frac{d^{(r-1)}(z)}{d z^{r-1}} \frac{N^{\prime}}{\left(z-p_{1}\right)\left(z-p_{2}\right), \ldots,\left(z-p_{n-r}\right)\left(z-p_{r}\right)^{r}}\left(z-p_{r}\right)^{r}\right|_{z=p_{1}} .
\end{aligned}
$$

Then, we can obtain

$$
\begin{aligned}
& k_{1}=k_{1}^{\prime}, k_{2}=k_{2}^{\prime}, \ldots, k_{n-r}=k_{n-r}^{\prime}, \\
& A_{r}^{\prime}=A_{r}, A_{r-1}^{\prime}=A_{r-1}, \ldots A_{1}^{\prime}=A_{1},
\end{aligned}
$$

that is, the long division operation truly is not necessary. The inverse transformation of $F(z)$ is 


$$
\begin{aligned}
f(k)= & \left(\frac{a_{n}}{b_{n}}\right) \delta(k)+k_{1}\left(p_{1}\right)^{k-1} u_{s}(k-1)+k_{2}\left(p_{2}\right)^{k-1} u_{s}(k-1)+\cdots+k_{n-r}\left(p_{n-r}\right)^{k-1} u_{s}(k-1) \\
& +A_{1}\left(p_{r}\right)^{k-1} u_{s}(k-1)+A_{2}\left(\frac{1}{1 !}\right)(k-1)\left(p_{r}\right)^{k-2} u_{s}(k-1)+\cdots \\
& +A_{r}\left(\frac{1}{(r-1) !}\right)(k-1)(k-2) \cdots(k-1-(r-2))\left(p_{r}\right)^{k-1-(r-1)} u_{s}(k-1) \\
= & \left(\frac{a_{n}}{b_{n}}\right) \delta(k)+\left.k_{1}\left(p_{1}\right)^{k} u_{s}(k)\right|_{k=k-1}+\left.k_{2}\left(p_{2}\right)^{k} u_{s}(k)\right|_{k=k-1}+\cdots+\left.k_{n-r}\left(p_{n-r}\right)^{k} u_{s}(k)\right|_{k=k-1} \\
& +\left.A_{1}\left(p_{r}\right)^{k} u_{s}(k)\right|_{k=k-1}+\left.A_{2}\left(\frac{1}{1 !}\right)(k-1)\left(p_{r}\right)^{k-2} u_{s}(k)\right|_{k=k-1}+\cdots \\
& +A_{r}\left(\frac{1}{(r-1) !}\right)(k)(k-1), \ldots,\left.(k-(r-2))\left(p_{r}\right)^{k-(r-1)} u_{s}(k)\right|_{k=k-1} .
\end{aligned}
$$

Proof. Applying Theorem 1 yields Then, $k_{1}=k_{1}^{\prime}, k_{2}=k_{2}^{\prime}, \ldots, k_{n-r}=k_{n-r}^{\prime}$. From (18) and (20), we get

$$
N(z)=\left(\frac{a_{n}}{b_{n}}\right)\left(z-p_{1}\right)\left(z-p_{2}\right) \cdots\left(z-p_{n-r}\right)\left(z-p_{r}\right)^{r}+N^{\prime}(z) \text {. }
$$

$$
\begin{aligned}
& A_{r}^{\prime}=\left.\frac{1}{0 !} \frac{d^{0}}{d z^{0}} \frac{N^{\prime}(z)}{\left(z-p_{1}\right)\left(z-p_{2}\right), \ldots,\left(z-p_{n-r}\right)\left(z-p_{r}\right)^{r}}\left(z-p_{r}\right)^{r}\right|_{z=p_{r}} \\
& =\left.\frac{1}{0 !} \frac{d^{0}}{d z^{0}} \frac{\left(a_{n} / b_{n}\right)\left(z-p_{1}\right)\left(z-p_{2}\right), \ldots,\left(z-p_{n-r}\right)\left(z-p_{r}\right)^{r}+N \prime(z)}{\left(z-p_{1}\right)\left(z-p_{2}\right), \ldots,\left(z-p_{n-r}\right)\left(z-p_{r}\right)^{r}}\left(z-p_{r}\right)^{r}\right|_{z=p_{r}} \\
& =\left.\frac{1}{0 !} \frac{d^{0}}{d z^{0}} \frac{N(z)}{\left(z-p_{1}\right)\left(z-p_{2}\right), \ldots,\left(z-p_{n-r}\right)\left(z-p_{r}\right)^{r}}\left(z-p_{r}\right)^{r}\right|_{z=p_{r}}=A_{r}, \\
& A_{r-1}^{\prime}=\left.\frac{1}{1 !} \frac{d^{1}}{d z^{1}} \frac{N^{\prime}(z)}{\left(z-p_{1}\right)\left(z-p_{2}\right), \ldots,\left(z-p_{n-r}\right)\left(z-p_{r}\right)^{r}}\left(z-p_{r}\right)^{r}\right|_{z=p_{r}} \\
& =\left.\frac{1}{1 !} \frac{d^{1}}{d z^{1}} \frac{\left(a_{n} / b_{n}\right)\left(z-p_{1}\right)\left(z-p_{2}\right), \ldots,\left(z-p_{n-r}\right)\left(z-p_{r}\right)^{r}+N \prime(z)}{\left(z-p_{1}\right)\left(z-p_{2}\right), \ldots,\left(z-p_{n-r}\right)\left(z-p_{r}\right)^{r}}\left(z-p_{r}\right)^{r}\right|_{z=p_{r}} \\
& =\left.\frac{1}{1 !} \frac{d^{1}}{d z^{1}} \frac{N(z)}{\left(z-p_{1}\right)\left(z-p_{2}\right), \ldots,\left(z-p_{n-r}\right)\left(z-p_{r}\right)^{r}}\left(z-p_{r}\right)^{r}\right|_{z=p_{r}}=A_{r-1}, \\
& A_{1}^{\prime}=\left.\frac{1}{(r-1) !} \frac{d^{(r-1)}}{d z^{r-1}} \frac{N^{\prime}(z)}{\left(z-p_{1}\right)\left(z-p_{2}\right), \ldots,\left(z-p_{n-r}\right)\left(z-p_{r}\right)^{r}}\left(z-p_{r}\right)^{r}\right|_{z=p_{r}} \\
& =\left.\frac{1}{(r-1) !} \frac{d^{(r-1)}}{d z^{r-1}} \frac{\left(a_{n} / b_{n}\right)\left(z-p_{1}\right)\left(z-p_{2}\right), \ldots,\left(z-p_{n-r}\right)\left(z-p_{r}\right)^{r}+N^{\prime}(z)}{\left(z-p_{1}\right)\left(z-p_{2}\right), \ldots,\left(z-p_{n-r}\right)\left(z-p_{r}\right)^{r}}\left(z-p_{r}\right)^{r}\right|_{z=p_{r}} \\
& =\left.\frac{1}{(r-1) !} \frac{d^{(r-1)}}{d z^{r-1}} \frac{N(z)}{\left(z-p_{1}\right)\left(z-p_{2}\right), \ldots,\left(z-p_{n-r}\right)\left(z-p_{r}\right)^{r}}\left(z-p_{r}\right)^{r}\right|_{z=p_{r}}=A_{1} .
\end{aligned}
$$


Define

$$
G(z)=\frac{1}{(z-p)^{n+1}} .
$$

Utilizing the inverse $z$-transformation theorem of Cauchy residue calculus gets

$$
g(k)=\text { Residues }\left[\frac{1}{(z-p)^{n+1}}\left(z^{k-1}\right)\right] \text {. }
$$

For nonpositive values $k$,

$$
\begin{aligned}
g(k)= & \text { Residues }\left[\frac{1}{(z-p)^{n+1} z^{1-k}}\right]=\underset{z=p}{\operatorname{Residue}} \frac{1}{(z-p)^{n+1} z^{1-k}}+\underset{z=0}{\operatorname{Residue}} \frac{1}{(z-p)^{n+1} z^{1-k}} \\
= & \left.\frac{1}{n !} \frac{\mathrm{d}^{(n)}}{\mathrm{d} z^{(n)}} \frac{1}{z^{1-k}}\right|_{z=p}+\left.\frac{1}{(-k) !} \frac{\mathrm{d}^{(-k)}}{\mathrm{d} z^{(-k)}} \frac{1}{(z-p)^{n+1}}\right|_{z=0} \\
= & \frac{1}{n !}(k-(1))(k-(2)), \ldots,(k-(n-1))(k-n)(p)^{k-n-1} \\
& +(-1)^{-n-1} \frac{1}{(-k) !}(n+(1))(n+(2)), \ldots,(n+(-k-1))(n-k)(p)^{k-n-1}=0 .
\end{aligned}
$$

For positive values $k$,

$$
\begin{aligned}
g(k) & =\text { Residues }\left[\frac{z^{k-1}}{(z-p)^{n+1}}\right]=\left[\operatorname{Residue}_{z=p} \frac{z^{k-1}}{(z-p)^{n+1}}\right] \\
& =\left.\frac{1}{n !} \frac{\mathrm{d}^{(n)}}{\mathrm{d} z^{(n)}}(z)^{k-1}\right|_{z=p}=\left(\frac{1}{n !}\right)(k-1)(k-2), \ldots,(k-(n-1))(k-n)(p)^{k-n-1} \\
& =\left(\frac{1}{n !}\right)(k-1)(k-2), \ldots,(k-(n-1))(k-n)(p)^{k-n-1} .
\end{aligned}
$$

Therefore, from equation (19), we get the inverse z-transformation of $F(z)$ as

$$
\begin{aligned}
f(k)= & \left(\frac{a_{n}}{b_{n}}\right) \delta(k)+k_{1}\left(p_{1}\right)^{k-1} u_{s}(k-1) \\
& +k_{2}\left(p_{2}\right)^{k-1} u_{s}(k-1)+\cdots+k_{n-r}\left(p_{n-r}\right)^{k-1} u_{s}(k-1) \\
& +A_{1}\left(p_{r}\right)^{k-1} u_{s}(k-1)+A_{2}\left(\frac{1}{1 !}\right)(k-1)\left(p_{r}\right)^{k-2} u_{s}(k-1)+\cdots+ \\
& A_{r}\left(\frac{1}{(r-1) !}\right)(k-1)(k-2), \ldots,(k-1-(r-2))\left(p_{r}\right)^{k-1-(r-1)} u_{s}(k-1) .
\end{aligned}
$$


Theorem 3. If a rational function $F(z)$ is given with complex conjugate poles, where the numerator and the denominator are in the same power,

$$
\begin{aligned}
F(z) & =\frac{a_{n} z^{n}+a_{n-1} z^{n-1}+\cdots+a_{1} z^{1}+a_{0}}{b_{n} z^{n}+b_{n-1} z^{n-1}+\cdots+b_{1} z^{1}+b_{0}}=\frac{\left(a_{n} / b_{n}\right) z^{n}+\left(a_{n-1} / b_{n}\right) z^{n-1}+\cdots+\left(a_{1} / b_{n}\right) z^{1}+\left(a_{0} / b_{n}\right)}{z^{n}+\left(b_{n-1} / b_{n}\right) z^{n-1}+\cdots+\left(b_{1} / b_{n}\right) z^{1}+\left(b_{0} / b_{n}\right)} \\
& \equiv \frac{\left(a_{n} / b_{n}\right) z^{n}+\left(a_{n-1} / b_{n}\right) z^{n-1}+\cdots+\left(a_{1} / b_{n}\right) z^{1}+\left(a_{0} / b_{n}\right)}{\left(z-p_{1}\right)\left(z-p_{2}\right), \ldots,\left(z-p_{n-2}\right)\left(z^{2}+2 \xi \omega_{n} z+\omega_{n}^{2}\right)} \\
& \equiv \frac{N(z)}{\left(z-p_{1}\right)\left(z-p_{2}\right), \ldots,\left(z-p_{n-2}\right)\left(z^{2}+2 \xi \omega_{n} z+\omega_{n}^{2}\right)} \\
N(z) & \equiv\left(\frac{a_{n}}{b_{n}}\right) z^{n}+\left(\frac{a_{n-1}}{b_{n}}\right) z^{n-1}+\cdots+\left(\frac{a_{1}}{b_{n}}\right) z^{1}+\left(\frac{a_{0}}{b_{n}}\right)
\end{aligned}
$$

then, we can obtain

$$
F(z)=\left(\frac{a_{n}}{b_{n}}\right)+\left[\frac{k_{1}}{z-p_{1}}+\frac{k_{2}}{z-p_{2}}+\cdots+\frac{k_{n-2}}{z-p_{n-2}}+\left(\frac{A z+B}{z^{2}+2 \xi \omega_{n} z+\omega_{n}^{2}}\right)\right]
$$

where $a_{n} / b_{n}$ is obtained by directly observing the ratio of the highest order coefficient of the numerator and the highest order coefficient of the denominator, and the parameters of the partial fraction will be shown as follows without the long division operation:

$$
\begin{aligned}
k_{1}= & \left.\frac{N(z)}{\left(z-p_{1}\right)\left(z-p_{2}\right), \ldots,\left(z-p_{n-2}\right)\left(z^{2}+2 \xi \omega_{n} z+\omega_{n}^{2}\right)}\left(z-p_{1}\right)\right|_{z=p_{1}}, \\
k_{2}= & \left.\frac{N(z)}{\left(z-p_{1}\right)\left(z-p_{2}\right), \ldots,\left(z-p_{n-2}\right)\left(z^{2}+2 \xi \omega_{n} z+\omega_{n}^{2}\right)}\left(z-p_{2}\right)\right|_{z=p_{2}}, \\
k_{n-2}= & \left.\frac{N(z)}{\left(z-p_{1}\right)\left(z-p_{2}\right), \ldots,\left(z-p_{n-2}\right)\left(z^{2}+2 \xi \omega_{n} z+\omega_{n}^{2}\right)}\left(z-p_{n-2}\right)\right|_{z=p_{n-2}}, \\
B= & {\left[\frac{\left(a_{0} / b_{n}\right)}{\left.\left(-p_{1}\right) \cdots\left(-p_{n-2}\right)\right]-\left[\left(\frac{a_{n}}{b_{n}}\right)-\frac{k_{1}}{p_{1}}-\frac{k_{2}}{p_{2}}-\cdots-\frac{k_{n-2}}{p_{n-2}}\right] \omega_{n}^{2},}\right.} \\
A= & {\left[\frac{\left(a_{n} / b_{n}\right)+\left(a_{n-1} / b_{n}\right)+\cdots+\left(a_{1} / b_{n}\right)+\left(a_{0} / b_{n}\right)}{\left(1-p_{1}\right) \cdots\left(1-p_{n-2}\right)}\right] } \\
& -\left[\frac{a_{n}}{b_{n}}+\frac{k_{1}}{1-p_{1}}+\cdots+\frac{k_{n-2}}{1-p_{n-2}}+\frac{B}{1+2 \xi \omega_{n}+\omega_{n}^{2}}\right]\left(1+2 \xi \omega_{n}+\omega_{n}^{2}\right) .
\end{aligned}
$$


The inverse transformation of $F(z)$ is

$$
\begin{aligned}
f(k)= & \left(\frac{a_{n}}{b_{n}}\right) \delta(k)+k_{1}\left(p_{1}\right)^{k-1} u_{s}(k-1)+k_{2}\left(p_{2}\right)^{k-1} u_{s}(k-1)+\cdots+k_{n-2}\left(p_{n-2}\right)^{k-1} u_{s}(k-1) \\
& +A\left(\omega_{n}\right)^{k-1} \cos ((k-1) \theta) u_{s}(k-1)+\left(\frac{B-A \xi \omega_{n}}{\sqrt{\omega_{n}^{2}-\xi^{2} \omega_{n}^{2}}}\right)\left(\omega_{n}\right)^{k-1} \sin ((k-1) \theta) u_{s}(k-1) \\
= & a_{n} \delta(k)+\left.k_{1}\left(p_{1}\right)^{k} u_{s}(k)\right|_{k=k-1}+\left.k_{2}\left(p_{2}\right)^{k} u_{s}(k)\right|_{k=k-1}+\cdots+\left.k_{n-2}\left(p_{n-2}\right)^{k} u_{s}(k)\right|_{k=k-1} \\
& +\left.A\left(\omega_{n}\right)^{k} \cos ((k) \theta) u_{s}(k)\right|_{k=k-1}+\left(\frac{B-A \xi \omega_{n}}{\sqrt{\omega_{n}^{2}-\xi^{2} \omega_{n}^{2}}}\right)\left(\omega_{n}\right)^{k} \sin ((k) \theta) u_{s}(k)_{k=k-1}, \\
\theta= & \tan ^{-1}\left(\frac{\sqrt{\omega_{n}^{2}-\xi^{2} \omega_{n}^{2}}}{\xi \omega_{n}}\right) .
\end{aligned}
$$

Proof. From Theorem 1, applying immediately the traditional partial fraction approach without the long division operation yields

$$
\begin{gathered}
F(z)=\left(\frac{a_{n}}{b_{n}}\right)+\left[\frac{k_{1}}{z-p_{1}}+\frac{k_{2}}{z-p_{2}}+\cdots+\frac{k_{n-2}}{z-p_{n-2}}+\left(\frac{A z+B}{z^{2}+2 \xi \omega_{n} z+\omega_{n}^{2}}\right)\right], \\
k_{1}=\left.\frac{N(z)}{\left(z-p_{1}\right)\left(z-p_{2}\right), \ldots,\left(z-p_{n-2}\right)\left(z^{2}+2 \xi \omega_{n} z+\omega_{n}^{2}\right)}\left(z-p_{1}\right)\right|_{z=p_{1}}, \\
k_{2}=\left.\frac{N(z)}{\left(z-p_{1}\right)\left(z-p_{2}\right), \ldots,\left(z-p_{n-2}\right)\left(z^{2}+2 \xi \omega_{n} z+\omega_{n}^{2}\right)}\left(z-p_{2}\right)\right|_{z=p_{2}}, \\
k_{n-2}=\left.\frac{N(z)}{\left(z-p_{1}\right)\left(z-p_{2}\right), \ldots,\left(z-p_{n-2}\right)\left(z^{2}+2 \xi \omega_{n} z+\omega_{n}^{2}\right)}\left(z-p_{n-2}\right)\right|_{z=p_{n-2}} .
\end{gathered}
$$

Substituting $z=0$ and $z=1$ individually into (32) and (34) gets

$$
\begin{aligned}
& \frac{\left(a_{0} / b_{n}\right)}{\left(-p_{1}\right), \ldots,\left(-p_{n-2}\right) \omega_{n}^{2}}=\left(\frac{a_{n}}{b_{n}}\right)-\frac{k_{1}}{p_{1}}-\frac{k_{2}}{p_{2}}-\cdots-\frac{k_{n-2}}{p_{n-2}}+\frac{B}{\omega_{n}^{2}} \\
& \frac{\left(a_{n} / b_{n}\right)+\left(a_{n-1} / b_{n}\right)+\cdots+\left(a_{1} / b_{n}\right)+\left(a_{0} / b_{n}\right)}{\left(1-p_{1}\right), \ldots,\left(1-p_{n-2}\right)\left(1+2 \xi \omega_{n}+\omega_{n}^{2}\right)} \\
& =\frac{a_{n}}{b_{n}}+\frac{k_{1}}{1-p_{1}}+\cdots+\frac{k_{n-2}}{1-p_{n-2}}+\frac{A+B}{1+2 \xi \omega_{n}+\omega_{n}^{2}} .
\end{aligned}
$$

$$
B=\left[\frac{\left(a_{0} / b_{n}\right)}{\left(-p_{1}\right), \ldots,\left(-p_{n-2}\right)}\right]
$$

$$
\begin{aligned}
& -\left[\left(\frac{a_{n}}{b_{n}}\right)-\frac{k_{1}}{p_{1}}-\frac{k_{2}}{p_{2}}-\cdots-\frac{k_{n-2}}{p_{n-2}}\right] \omega_{n}^{2}, \\
A= & {\left[\frac{\left(a_{n} / b_{n}\right)+\left(a_{n-1} / b_{n}\right)+\cdots+\left(a_{1} / b_{n}\right)+\left(a_{0} / b_{n}\right)}{\left(1-p_{1}\right) \cdots\left(1-p_{n-2}\right)}\right] } \\
& -\left[\frac{a_{n}}{b_{n}}+\frac{k_{1}}{1-p_{1}}+\cdots+\frac{k_{n-2}}{1-p_{n-2}}+\frac{B}{1+2 \xi \omega_{n}+\omega_{n}^{2}}\right] \\
& \cdot\left(1+2 \xi \omega_{n}+\omega_{n}^{2}\right) .
\end{aligned}
$$

Then,

From (37), we get 


$$
F(z)=\frac{a_{n}}{b_{n}}+\left[\frac{k_{1}}{z-p_{1}}+\frac{k_{2}}{z-p_{2}}+\cdots+\frac{k_{n-2}}{z-p_{n-2}}+\left(\frac{A z+B}{z^{2}+2 \xi \omega_{n} z+\omega_{n}^{2}}\right)\right] .
$$

Utilizing the simple algebraic operations yields

$$
F(z)=\frac{a_{n}}{b_{n}}+\left[\frac{k_{1}}{z-p_{1}}+\frac{k_{2}}{z-p_{2}}+\cdots+\frac{k_{n-2}}{z-p_{n-2}}+\left(\frac{A\left(z+\xi \omega_{n}\right)+\left(B-A \xi \omega_{n} / \sqrt{\omega_{n}^{2}-\xi^{2} \omega_{n}^{2}}\right) \sqrt{\omega_{n}^{2}-\xi^{2} \omega_{n}^{2}}}{\left(z+\xi \omega_{n}\right)^{2}+\left(\sqrt{\omega_{n}^{2}-\xi^{2} \omega_{n}^{2}}\right)^{2}}\right)\right]
$$

Define

$$
\begin{aligned}
& S(z)=\frac{\omega}{(z-p)^{2}+\omega^{2}}, \\
& C(z)=\frac{(z-p)}{(z-p)^{2}+\omega^{2}} .
\end{aligned}
$$

$$
\begin{aligned}
& S(z)=\frac{\omega}{(z-p)^{2}+\omega^{2}}=\frac{-1 / 2 i}{z-(p+j \omega)}+\frac{+1 / 2 i}{z-(a-j \omega)}, \\
& C(z)=\frac{(z-p)}{(z-p)^{2}+\omega^{2}}=\frac{1 / 2}{z-(p+j \omega)}+\frac{1 / 2}{z-(a-j \omega)} .
\end{aligned}
$$

According to Theorem 1 and the Euler's formula, the

Applying the factor decomposition and the partial inverse $z$-transformations of $C(z)$ and $S(z)$ are given by fraction expansion gets

$$
\begin{aligned}
& S(k)=\left.\left[\left(-\frac{1}{2} i\right)(p+j \omega)^{k}+\left(\frac{1}{2} i\right)(p-j \omega)^{k}\right] u_{s}(k)\right|_{k=k-1} \\
& =\left.\left[\left(-\frac{1}{2} i\right)\left(\sqrt{p^{2}+\omega^{2}} e^{j(\angle(p+j \omega))}\right)^{k}+\left(\frac{1}{2} i\right)\left(\sqrt{p^{2}+\omega^{2}} e^{-j(\angle(p+j \omega))}\right)^{k}\right] u_{s}(k)\right|_{k=k-1} \\
& =\left.\left(-\frac{1}{2} i\right)\left(\sqrt{p^{2}+\omega^{2}}\right)^{k}\left[e^{j((k)(\angle(p+j \omega)))}-e^{j(-(k)(\angle(p+j \omega)))}\right] u_{s}(k)\right|_{k=k-1} \\
& =\left.\left(-\frac{1}{2} i\right)\left(\sqrt{p^{2}+\omega^{2}}\right)^{k}[2 i \sin ((k) \theta)] u_{s}(k)\right|_{k=k-1} \\
& =\left.\left(\sqrt{p^{2}+\omega^{2}}\right)^{k}[\sin ((k) \theta)] u_{s}(k)\right|_{k=k-1} \\
& C(k)=\left.\left[\frac{1}{2}(p+j \omega)^{k}+\frac{1}{2}(p-j \omega)^{k}\right] u_{s}(k)\right|_{k=k-1} \\
& =\left.\left[\frac{1}{2}\left(\sqrt{p^{2}+\omega^{2}} e^{j(\angle(p+j \omega))}\right)^{k}+\frac{1}{2}\left(\sqrt{p^{2}+\omega^{2}} e^{-j(\angle(p+j \omega))}\right)^{k}\right] u_{s}(k)\right|_{k=k-1} \\
& =\left.\frac{1}{2}\left(\sqrt{p^{2}+\omega^{2}}\right)^{k}\left[e^{j((k)(\angle(p+j \omega)))}+e^{j(-(k)(\angle(p+j \omega)))}\right] u_{s}(k)\right|_{k=k-1} \\
& =\left.\frac{1}{2}\left(\sqrt{p^{2}+\omega^{2}}\right)^{k}[2 \cos ((k) \theta)] u_{s}(k)\right|_{k=k-1} \\
& =\left.\left(\sqrt{p^{2}+\omega^{2}}\right)^{k}[\cos ((k) \theta)] u_{s}(k)\right|_{k=k-1} \text {. }
\end{aligned}
$$


Therefore, from equation (42), the inverse $z$-transformation of $F(z)$ is given as

$$
\begin{aligned}
f(k)= & \left(\frac{a_{n}}{b_{n}}\right) \delta(k)+k_{1}\left(p_{1}\right)^{k-1} u_{s}(k-1) \\
& +k_{2}\left(p_{2}\right)^{k-1} u_{s}(k-1)+\cdots \\
& +k_{n-2}\left(p_{n-2}\right)^{k-1} u_{s}(k-1) \\
& +A\left(\omega_{n}\right)^{k-1} \cos ((k-1) \theta) u_{s}(k-1) \\
& +\left(\frac{B-A \xi \omega_{n}}{\sqrt{\omega_{n}^{2}-\xi^{2} \omega_{n}^{2}}}\right)\left(\omega_{n}\right)^{k-1} \sin ((k-1) \theta) u_{s}(k-1)
\end{aligned}
$$

\section{Comparative Examples}

In this section, some comparative examples will be proposed to show that the direct method provided in this study is better than that of the existing traditional approaches.

Example 1. An economic cybernetic transfer function is known as

$$
H(\mathrm{z}) \equiv \frac{F(z)}{R(z)}=\frac{\left(z^{5}+2\right)(z-1)}{z^{2}(z-2)(z-4)(z-6)(z-8)} .
$$

Let us find the unit step response of the system. The input function of the system is given as

$$
R(z)=\frac{z}{z-1} \text {. }
$$

Then, the unit step response of the system is

$$
F(z)=\frac{z^{5}+2}{z^{1}(z-2)(z-4)(z-6)(z-8)} .
$$

Immediately we will use some traditional methods and our proposed direct method to solve it and make some comparisons between them.

\subsection{Traditional Method 1: Long-Division and Partial Fraction} Expansion Method. In order to use the long division and partial fraction expansion method, we have to expand all the polynomial factors as follows:

$$
F(z)=\frac{z^{5}+2}{z^{5}-20 z^{4}+140 z^{3}-400 z^{2}+384 z+0} .
$$

The following equation can be derived using long division:

$F(z)=1+\frac{20 z^{4}-140 z^{3}+400 z^{2}-384 z+2}{z^{5}-20 z^{4}+140 z^{3}-400 z^{2}+384 z+0}$.

That is,

$$
F(z)=1+\frac{20 z^{4}-140 z^{3}+400 z^{2}-384 z+2}{z^{1}(z-2)(z-4)(z-6)(z-8)} .
$$

Define

$$
F^{\prime}(z)=\frac{20 z^{4}-140 z^{3}+400 z^{2}-384 z+2}{z^{1}(z-2)(z-4)(z-6)(z-8)} .
$$

Applying the partial fraction method yields

$$
\begin{aligned}
F(z) & =1+\frac{A^{\prime}}{z}+\frac{B^{\prime}}{z-2}+\frac{C^{\prime}}{z-4}+\frac{D^{\prime}}{z-6}+\frac{E^{\prime}}{z-8}, \\
A^{\prime} & =\left.F^{\prime}(z)\right|_{z=0}=\frac{1}{(-2)(-4)(-6)(-8)}=\frac{1}{192}, \\
B^{\prime} & =\left.F^{\prime}(z-2)\right|_{z=2}=\frac{320-1120+1600-768+2}{(2)(-2)(-4)(-6)}=-\frac{17}{48}, \\
C^{\prime} & =\left.F^{\prime}(z-4)\right|_{z=4}=\frac{5120-8960+6400-1536+2}{(4)(2)(-2)(-4)}=\frac{513}{32}, \\
D^{\prime} & =\left.F^{\prime}(z-6)\right|_{z=6}=\frac{25920-30240+14400-2304+2}{(6)(4)(2)(-2)}=-\frac{3889}{48}, \\
E^{\prime} & =\left.F^{\prime}(z-8)\right|_{z=8}=\frac{81920-71680+25600-3072+2}{(8)(6)(4)(2)}=\frac{16385}{192} .
\end{aligned}
$$


Then,

$$
\begin{aligned}
F(z)= & +\frac{(1 / 192)}{z}+\frac{(-17 / 48)}{z-2} \\
& +\frac{(513 / 32)}{z-4}+\frac{(-3889 / 48)}{z-6}+\frac{(16385 / 192)}{z-8} .
\end{aligned}
$$

Finally, according to Theorem 1, the desired solution is

$$
\begin{aligned}
f(k)= & \delta(k)+\left(\frac{1}{192}\right) \delta(k-1) \\
& +\left(-\frac{17}{48}\right)(2)^{k-1} u_{s}(k-1) \\
& +\left(\frac{513}{32}\right)(4)^{k-1} u_{s}(k-1)+\left(-\frac{3889}{48}\right)(6)^{k-1} u_{s}(k-1)
\end{aligned}
$$

that is, $f(0)=1, f(1)=20$, and

$$
\begin{aligned}
\left.f(k)\right|_{k \geq 2}= & \left(-\frac{17}{48}\right)(2)^{k-1}+\left(\frac{513}{32}\right)(4)^{k-1} \\
& +\left(-\frac{3889}{48}\right)(6)^{k-1}+\left(\frac{16385}{192}\right)(8)^{k-1} .
\end{aligned}
$$

It is obvious to see that the shortcomings of the long division and partial fraction method include three tedious operations: the multiplication of all polynomial factors, the long division of two complex polynomials, and the derivations of the complicated partial fraction variables.

3.2. Traditional Method 2: Divided by $z$ and Partial Fraction Expansion Method. First divide $F(z)$ by $z$ as

$$
\frac{F(z)}{z}=\frac{z^{5}+2}{z^{2}(z-2)(z-4)(z-6)(z-8)} .
$$

Using the partial fraction method gives

$$
\begin{aligned}
\frac{F(z)}{z} & =\left[\frac{A_{2}}{z}+\frac{B_{2}}{z^{2}}\right]+\frac{C_{2}}{z-2}+\frac{C_{3}}{z-4}+\frac{C_{4}}{z-6}+\frac{C_{5}}{z-8}, \\
A_{2} & =\left.\frac{1}{1 !} \frac{\mathrm{d}}{\mathrm{d} z}\left[\frac{z^{5}+2}{(z-2)(z-4)(z-6)(z-8)}\right]\right|_{z=0} \\
& =\left.\frac{1}{1 !} \frac{d}{d z}\left[\frac{z^{5}+2}{z^{4}-20 z^{3}+140 z^{2}-400 z^{1}+384}\right]\right|_{z=0} \\
& =\left.\left[\frac{\left(N_{1}\right)\left(5 z^{4}\right)-\left(z^{5}+2\right)\left(N_{2}\right)}{\left(N_{1}\right)^{2}}\right]\right|_{z=0}=\frac{25}{4608}, \\
N_{1} & \equiv z^{4}-20 z^{3}+140 z^{2}-400 z^{1}+384, \\
N_{2} & \equiv 4 z^{3}-60 z^{2}+280 z^{1}-400, \\
B_{2} & =\left.\frac{1}{0 !} \frac{\mathrm{d}}{\mathrm{d} z^{0}}\left[\frac{z^{5}+2}{z^{4}-20 z^{3}+140 z^{2}-400 z^{1}+384}\right]\right|_{z=0}=\frac{1}{192}, \\
C_{2} & =\left.\left(\frac{z^{5}+2}{z^{2}(z-2)(z-4)(z-6)(z-8)}\right)(z-2)\right|_{z=2}=-\frac{177}{96}, \\
C_{3} & =\left.\left(\frac{z^{5}+2}{z^{2}(z-2)(z-4)(z-6)(z-8)}\right)(z-4)\right|_{z=4}=\frac{513}{128}, \\
C_{4} & =\left.\left(\frac{z^{5}+2}{z^{2}(z-2)(z-4)(z-6)(z-8)}\right)(z-6)\right|_{z=6}=-\frac{3889}{288}, \\
C_{5} & =\left.\left(\frac{z^{2}(z-2)(z-4)(z-6)(z-8)}{z^{2}}\right)(z-8)\right|_{z=8}=\frac{16385}{1536} .
\end{aligned}
$$


Thus,

$$
\begin{aligned}
F(z)= & \frac{25}{4608}+\left(\frac{1}{192}\right) \frac{1}{z}+\left(-\frac{17}{96}\right) \frac{z}{z-2}+\left(\frac{513}{128}\right) \frac{z}{z-4} \\
& +\left(-\frac{3889}{288}\right) \frac{z}{z-6}+\left(\frac{16385}{1536}\right) \frac{z}{z-8} .
\end{aligned}
$$

Applying Theorem 1 gives the desired solution as

$$
\begin{aligned}
f(k)= & \frac{25}{4608} \delta(k)+\left(\frac{1}{192}\right) \delta(k-1)+\left(-\frac{17}{96}\right)(2)^{k} \\
& +\left(\frac{513}{128}\right)(4)^{k}+\left(-\frac{3889}{288}\right)(6)^{k}+\left(\frac{16385}{1536}\right)(8)^{k},
\end{aligned}
$$

that is, $f(0)=1, f(1)=20$, and

$$
\begin{aligned}
\left.f(k)\right|_{k \geq 2}= & \left(-\frac{17}{96}\right)(2)^{k}+\left(\frac{513}{128}\right)(4)^{k} \\
& +\left(-\frac{3889}{288}\right)(6)^{k}+\left(\frac{16385}{1536}\right)(8)^{k} .
\end{aligned}
$$

It is obvious to see that this specific $F(z)$ with $z$-factor will increase the burden of hand-calculation for including two tedious operations: the multiplication of all polynomial factors and the differentiation of two complex polynomials.
3.3. Traditional Method 3: Inversion Integral Method. According to the inversion integral method, we get the invers $z$-transformation of $F(z)$ as

$$
\begin{aligned}
f(k) & =\text { Residues }\left[\frac{\left(z^{5}+2\right) z^{k-1}}{z^{1}(z-2)(z-4)(z-6)(z-8)}\right] \\
& =\text { Residues }\left[\frac{\left(z^{5}+2\right) z^{k-2}}{(z-2)(z-4)(z-6)(z-8)}\right] .
\end{aligned}
$$

For the case of $k=0$, the desired solution is

$$
f(k)=\text { Residues }\left[\frac{\left(z^{5}+2\right)}{z^{2}(z-2)(z-4)(z-6)(z-8)}\right]
$$

where

$$
M_{1}(z) \equiv \frac{\left(z^{5}+2\right)}{z^{2}(z-2)(z-4)(z-6)(z-8)} .
$$

Consequently,

$$
\begin{aligned}
f(k)= & \underset{z=0}{\operatorname{Residue}}\left[M_{1}(z)\right]+\underset{z=2}{\operatorname{Residue}}\left[M_{1}(z)\right] \\
& +\underset{z=4}{\operatorname{Residue}}\left[M_{1}(z)\right] \\
& +\underset{z=6}{\operatorname{Residue}}\left[M_{1}(z)\right]+\underset{z=8}{\operatorname{Residue}}\left[M_{1}(z)\right],
\end{aligned}
$$

where

$$
\begin{aligned}
& \underset{z=0}{\operatorname{Residue}}\left[M_{1}(z)\right]=\left.\frac{1}{1 !} \frac{\mathrm{d}}{\mathrm{d} z}\left[\frac{\left(z^{5}+2\right)}{(z-2)(z-4)(z-6)(z-8)}\right]\right|_{z=0} \\
& =\left.\frac{1}{1 !} \frac{\mathrm{d}}{\mathrm{d} z}\left[\frac{z^{5}+2}{z^{4}-20 z^{3}+140 z^{2}-400 z^{1}+384}\right]\right|_{z=0} \\
& =\left.\left[\frac{\left(N_{1}\right)\left(5 z^{4}\right)-\left(z^{5}+2\right)\left(N_{2}\right)}{\left(N_{1}\right)^{2}}\right]\right|_{z=0}=\frac{25}{4608} \\
& N_{1} \equiv z^{4}-20 z^{3}+140 z^{2}-400 z^{1}+384 \text {, } \\
& N_{2} \equiv 4 z^{3}-60 z^{2}+280 z^{1}-400, \\
& \underset{z=2}{\operatorname{Residue}}\left[M_{1}(z)\right]=\left.\left(\frac{\left(z^{5}+2\right)}{z^{2}(z-2)(z-4)(z-6)(z-8)}\right)(z-2)\right|_{z=2}=-\frac{17}{96}, \\
& \underset{z=4}{\operatorname{Residue}}\left[M_{1}(z)\right]=\left.\left(\frac{\left(z^{5}+2\right)}{z^{2}(z-2)(z-4)(z-6)(z-8)}\right)(z-4)\right|_{z=4}=\frac{513}{128}, \\
& \underset{z=6}{\operatorname{Residue}}\left[M_{1}(z)\right]=\left.\left(\frac{\left(z^{5}+2\right)}{z^{2}(z-2)(z-4)(z-6)(z-8)}\right)(z-6)\right|_{z=6}=-\frac{3889}{288}, \\
& \underset{z=8}{\operatorname{Residue}}\left[M_{1}(z)\right]=\left.\left(\frac{\left(z^{5}+2\right)}{z^{2}(z-2)(z-4)(z-6)(z-8)}\right)(z-8)\right|_{z=8}=\frac{16385}{1536} .
\end{aligned}
$$


For the case of $k=0$, the desired solution is derived as

$$
\begin{aligned}
f(0)= & \underset{z=0}{\operatorname{Residue}}\left[M_{1}(z)\right]+\underset{z=2}{\operatorname{Residue}}\left[M_{1}(z)\right] \\
& +\underset{z=4}{\operatorname{Residue}}\left[M_{1}(z)\right]+\underset{z=6}{\operatorname{Residue}}\left[M_{1}(z)\right] \\
& +\underset{z=8}{\operatorname{Residue}}\left[M_{1}(z)\right]=1 .
\end{aligned}
$$

Consider the case of $k=1$ as follows:

$$
\begin{aligned}
f(k) & =\text { Residues }\left[\frac{\left(z^{5}+2\right)}{(z-2)(z-4)(z-6)(z-8) z^{1}}\right] \\
& \equiv \operatorname{Residues}\left[M_{2}(z)\right],
\end{aligned}
$$

where

$$
M_{2}(z) \equiv \frac{\left(z^{5}+2\right)}{(z-2)(z-4)(z-6)(z-8) z^{1}}
$$

Thus,

$$
\begin{aligned}
f(k)= & \underset{z=2}{\operatorname{Residue}}\left[M_{2}(z)\right]+\underset{z=4}{\operatorname{Residue}}\left[M_{2}(z)\right] \\
& +\underset{z=6}{\operatorname{Residue}}\left[M_{2}(z)\right]+\underset{z=8}{\operatorname{Residue}}\left[M_{2}(z)\right] \\
& +\underset{z=0}{\operatorname{Residue}}\left[M_{2}(z)\right]=20 .
\end{aligned}
$$

For the case of $k \geq 2$, the inverse transformation of $F(z)$ is

$$
\begin{aligned}
f(k) & =\operatorname{Residues}\left[\frac{\left(z^{5}+2\right) z^{k-2}}{(z-2)(z-4)(z-6)(z-8)}\right] \\
& \equiv \operatorname{Residues}\left[M_{3}(z)\right],
\end{aligned}
$$

where

$$
M_{3}(z) \equiv \frac{\left(z^{5}+2\right) z^{k-2}}{(z-2)(z-4)(z-6)(z-8)} .
$$

Thus,

$$
\begin{aligned}
f(k)= & \underset{z=2}{\operatorname{Residue}}\left[M_{3}(z)\right]+\underset{z=4}{\operatorname{Residue}}\left[M_{3}(z)\right] \\
& +\underset{z=6}{\operatorname{Residue}}\left[M_{3}(z)\right]+\underset{z=8}{\operatorname{Residue}}\left[M_{3}(z)\right] \\
= & \left(-\frac{17}{96}\right)(2)^{k}+\left(\frac{514}{128}\right)(4)^{k} \\
& +\left(-\frac{3889}{288}\right)(6)^{k}+\left(\frac{16385}{1536}\right)(8)^{k} .
\end{aligned}
$$

It is easy to see that the disadvantages of the inversion integral method include two tedious operations: the multiplication of all polynomial factors and the differentiation of two complicated polynomials.

3.4. Traditional Method 4: Direct Long-Division Method. To apply the direct long-division method, we should expand all the factors.

$$
\begin{aligned}
F(z) & =\frac{z^{5}+2}{z(z-2)(z-4)(z-6)(z-8)} \\
& =\frac{z^{5}+2}{z^{5}-20 z^{4}+140 z^{3}-400 z^{2}+384 z+0} .
\end{aligned}
$$

The following equation can be derived using direct long division:

$$
\begin{aligned}
F(z) & =\frac{z^{5}+2}{z^{5}-20 z^{4}+140 z^{3}-400 z^{2}+384 z+0} \\
& =\frac{1+2 z^{-5}}{1-20 z^{-1}+140 z^{-2}-400 z^{-3}+384 z^{-4}+0} \\
& =1+20 z^{-1}+\cdots .
\end{aligned}
$$

Thus,

$$
\begin{aligned}
& f(0)=1, \\
& f(1)=20, \ldots .
\end{aligned}
$$

It is obvious to see that the direct long-division method only gives some finite terms of $F(k)$ and also needs two tedious operations: the multiplication of all polynomial factors and the long division of two polynomials.

3.5. Our Proposed Direct Method 5. To solve these shortcomings of the above existing traditional approaches, we directly use Theorem 1 to easily solve the discrete-time solution with shorter solving time than the traditional methods without the multiplication of all polynomial factors, the long division of two complex polynomials, the differentiation of two complex polynomials, and the derivations of the complex partial fraction variables as follows:

$$
F(z)=1+\frac{A}{z}+\frac{B}{z-2}+\frac{C}{z-4}+\frac{D}{z-6}+\frac{E}{z-8},
$$

where $A=\left.F(z)\right|_{z=0}=1 / 192 B=\left.F(z-2)\right|_{z=2}=-17 / 48 C=$ $\left.F(z-4)\right|_{z=4}=513 / 32 D=\left.F(z-6)\right|_{z=} \quad 6=-3889 / 48 E=F$ $\left.(z-8)\right|_{z=8}=16385 / 192$,

that is, 


$$
\begin{aligned}
F(z)= & 1+\frac{(1 / 192)}{z}+\frac{(-17 / 48)}{z-2}+\frac{(513 / 32)}{z-4} \\
& +\frac{(-3889 / 48)}{z-6}+\frac{(16385 / 192)}{z-8} .
\end{aligned}
$$

Consequently,

$$
\begin{aligned}
f(k)= & \delta(k)+\left(\frac{1}{192}\right) \delta(k-1) \\
& +\left(-\frac{17}{48}\right)(2)^{k-1} u_{s}(k-1) \\
& +\left(\frac{513}{32}\right)(4)^{k-1} u_{s}(k-1)+\left(-\frac{3889}{48}\right)(6)^{k-1} u_{s}(k-1) \\
& +\left(\frac{16385}{192}\right)(8)^{k-1} u_{s}(k-1),
\end{aligned}
$$

that is, $f(0)=1, f(1)=20$, and

$$
\begin{aligned}
\left.f(k)\right|_{k \geq 2}= & \left(-\frac{17}{48}\right)(2)^{k-1}+\left(\frac{513}{32}\right)(4)^{k-1}+\left(-\frac{3889}{48}\right)(6)^{k-1} \\
& +\left(\frac{16385}{192}\right)(8)^{k-1}
\end{aligned}
$$

Example 2. Let us solve the inverse $z$-transformation of

$$
F(\mathrm{z})=\frac{z^{3}}{(z+1)(z-2)^{2}},
$$

with the multiple poles $(z-2)$ case by using of the traditional long division method. We expand the denominator of $F(z)$ as follows:

$$
F(z)=\frac{z^{3}}{z^{3}-3 z^{2}+4} .
$$

Applying the long division method yields

$$
\begin{aligned}
F(z) & =\frac{z^{3}}{z^{3}-3 z^{2}+4}=1+\frac{3 z^{2}-4}{z^{3}-3 z^{2}+4} \\
& =1+\frac{3 z^{2}-4}{(z+1)(z-2)^{2}} .
\end{aligned}
$$

Then, we rewrite $F(z)$ into the partial fraction expansion form as follows:

$$
\begin{aligned}
F(z)= & 1+\frac{3 z^{2}-4}{(z+1)(z-2)^{2}}=1+\frac{-1 / 9}{(z+1)} \\
& +\frac{28 / 9}{(z-2)^{1}}+\frac{8 / 3}{(z-2)^{2}} .
\end{aligned}
$$

According to Theorems 1 and 2, the inverse $z$-transformation of $F(z)$ is given by

$$
\begin{aligned}
f(k)= & \delta(k)+(-1 / 9)(-1)^{k-1} u_{s}(k-1) \\
& +(28 / 9)(2)^{k-1} u_{s}(k-1) \\
& +(8 / 3)(k-1)(2)^{k-2} u_{s}(k-1)
\end{aligned}
$$

To be in comparison with the above traditional method, we easily apply the proposed Theorems 1 and 2 to get the following equation without using the traditional long division operation:

$$
F(\mathrm{z})=\frac{z^{3}}{(z+1)(z-2)^{2}}=1+\frac{-1 / 9}{(z+1)}+\frac{28 / 9}{(z-2)^{1}}+\frac{8 / 3}{(z-2)^{2}} .
$$

Then, we can immediately obtain the inverse $z$-transformation of $F(z)$ via Theorems 1 and 2 as follows:

$$
\begin{aligned}
f(k)= & \delta(k)+\left(-\frac{1}{9}\right)(-1)^{k-1} u_{s}(k-1) \\
& +\left(\frac{28}{9}\right)(2)^{k-1} u_{s}(k-1) \\
& +\left(\frac{8}{3}\right)(k-1)(2)^{k-2} u_{s}(k-1) .
\end{aligned}
$$

Example 3. Let us obtain the inverse $z$-transformation of

$$
F(\mathrm{z})=\frac{z^{3}}{(z+1)\left(z^{2}-2 z+5\right)}
$$

with the complex conjugate poles case by using of the traditional partial fractional expansion method [21]. Applying the long division method yields

$$
F(z)=1+\frac{z^{2}-3 z-5}{(z+1)\left(z^{2}-2 z+5\right)}
$$

We may expand $F(z)$ into partial fraction as follows: 


$$
F(z)=1+\frac{(-1 / 8)}{z+1}+\frac{(9 / 8) z+(-35 / 8)}{z^{2}-2 z+5}
$$

Noting that the two poles involved in the quadratic term of the last equation are complex conjugates, we rewrite $F(z)$ as follows:

$$
\begin{aligned}
F(\mathrm{z}) & =1+\left(z^{-1}\right) \frac{(-1 / 8) z}{z-1}+\left(\frac{9}{8}\right)\left(z^{-1}\right)\left[\frac{z^{2}-(2.23)(0.448) z}{z^{2}-1.2 z+0.5}+(-1.45) \frac{(2.23)(0.894) z}{z^{2}-1.2 z+0.5}\right] \\
& =1+\left(z^{-1}\right)\left(-\frac{1}{8}\right) \frac{z}{z-1}+\left(\frac{9}{8}\right)\left(z^{-1}\right)\left[\frac{z^{2}-0.999 z}{z^{2}-1.2 z+0.5}+(-1.45) \frac{1.993 z}{z^{2}-1.2 z+0.5}\right] .
\end{aligned}
$$

According to the $\mathrm{z}$-transformation table, the complicated shifting formulas of the sinusoidal function are given as

$$
\begin{aligned}
& z\left[e^{-a k T} \sin (\omega k T)\right]=\frac{z \cdot e^{-a T} \cdot \sin (\omega T)}{z^{2}-2 \cdot e^{-a T} \cdot z \cdot \cos (\omega T)+e^{-2 a T}} \\
& z\left[e^{-a k T} \cos (\omega k T)\right]=\frac{z^{2}-e^{-a T} \cdot z \cdot \cos (\omega T)}{z^{2}-2 \cdot e^{-a T} \cdot z \cdot \cos (\omega T)+e^{-2 a T}} .
\end{aligned}
$$

By identifying $e^{-2 a T}=5,2 \cdot e^{-a T} \cdot \cos (\omega T)=2$ and $\cos (\omega T)=0.448$ in this case, we have $\omega T=1.1(\mathrm{rad})$ and $\sin (\omega T)=0.894$. Hence, we obtain

$$
\begin{aligned}
& z^{-1}\left[\frac{z^{2}-0.999 z}{z^{2}-1.2 z+0.5}\right]=(2.23)^{k} \cos (1.1 k), \\
& z^{-1}\left[\frac{1.993 z}{z^{2}-1.2 z+0.5}\right]=(2.23)^{k} \sin (1.1 k),
\end{aligned}
$$

Thus, we have

$f(k)=\delta(k)+\left(-\frac{1}{8}\right) \cdot(-1)^{k-1} u_{s}(k-1)+\left(\frac{9}{8}\right)(2.23)^{k-1} \cos (1.1(k-1)) u_{s}(k-1)+\left(-\frac{13}{8}\right)(2.23)^{k-1} \sin (1.1(k-1)) u_{s}(k-1)$

that is,

$$
f(k)= \begin{cases}0, & k<0 \\ 1, & k=0 \\ +\left(-\frac{1}{8}\right) \cdot(-1)^{k-1}+\left(\frac{9}{8}\right)(2.23)^{k-1} \cos (1.1(k-1))+\left(-\frac{13}{8}\right)(2.23)^{k-1} \sin (1.1(k-1)), & k \geq 1 .\end{cases}
$$

To compare with the above traditional method, we easily apply the proposed Theorem 3 to solve the same problem. Using the simple algebraic operations gets

$$
\begin{aligned}
F(z) & =1+\frac{z^{2}-3 z-5}{(z+1)\left(z^{2}-2 z+5\right)}=1+\frac{(-1 / 8)}{z+1}+\frac{(9 / 8) z+(-35 / 8)}{z^{2}-2 z+5} \\
& =1+\frac{(-1 / 8)}{z+1}+\frac{(9 / 8)(z-1) z+(-13 / 8)(2)}{(z-1)^{2}+2^{2}}
\end{aligned}
$$


We immediately obtain

$$
\begin{aligned}
f(k)= & \delta(k)+\left(-\frac{1}{8}\right) \cdot(-1)^{k-1} u_{s}(k-1)+\left(\frac{9}{8}\right)(2.23)^{k-1} \cos (1.1(k-1)) u_{s}(k-1) \\
& +\left(-\frac{13}{8}\right)(2.23)^{k-1} \sin (1.1(k-1)) u_{s}(k-1)
\end{aligned}
$$

It is obvious to see that applying the complicated shifting formulas of the z-transformation table is not easy. On the contrary, a simple direct method that needs only easy algebraic operations has been proposed in this study for the case of complex conjugate poles.

\section{Economic Applications}

4.1. [Application 1]. Economic Cybernetics in Population Prediction. Suppose that country A has a population of 180 million in 2020 and B city of that country A has a population of 20 million. In $\mathrm{B}$ city, $8 \%$ of the population in the previous year move out to other parts of the country. In other parts of country A, $4 \%$ of the population in the previous year move into B city. Define the natural population growth rate as the difference between birth rate and mortality rate. Assume that the annual natural population growth rate is $2 \%$ and try to predict the population of B city. Suppose that the population of the $k$ th year for B city is $x 1(k)$, and the population of the country other than the B city is $x 2(k)$, then

$$
\begin{aligned}
x_{1}(k+1) & =(1+2 \%)\left[(1-8 \%) x_{1}(k)+4 \% x_{2}(k)\right], \\
x_{2}(k+1) & =(1+2 \%)\left[(8 \%) x_{1}(k)+(1-4 \%) x_{2}(k)\right], \\
x_{1}(0) & =2 \times 10^{7}, \\
x_{2}(0) & =18 \times 10^{7},
\end{aligned}
$$

that is,

$$
\begin{aligned}
X(k+1) & =A X(k), \\
X(0) & \equiv\left[\begin{array}{l}
x_{1}(0) \\
x_{2}(0)
\end{array}\right]=\left[\begin{array}{c}
2 \times 10^{7} \\
18 \times 10^{7}
\end{array}\right], \\
A & =\left[\begin{array}{ll}
0.9384 & 0.0408 \\
0.0816 & 0.9792
\end{array}\right] .
\end{aligned}
$$

Apply the z-transformation to solve the solution as follows:

$$
(z I-A)^{-1} z=\left[\begin{array}{cc}
\frac{z^{2}-0.9792 z}{(z-2.0392)(z-1.796)} & \frac{0.0408 z}{(z-2.0392)(z-1.796)} \\
\frac{0.0816 z}{(z-2.0392)(z-1.796)} & \frac{z^{2}-0.9384 z}{(z-2.0392)(z-1.796)}
\end{array}\right]
$$

In order to show the superiority of our proposed method, here, we first use the traditional method to solve it. For the traditional method $[20,21]$, the denominator and the numerator polynomial must be multiplied first to facilitate the long division operation because the denominator and the numerator have the same order.

$$
(z I-A)^{-1} z=\left[\begin{array}{cc}
\frac{z^{2}-0.9792 z}{z^{2}-3.8352 z+3.6624} & \frac{0.0408 z}{z^{2}-3.8352 z+3.6624} \\
\frac{0.0816 z}{z^{2}-3.8352 z+3.6624} & \frac{z^{2}-0.9384 z}{z^{2}-3.8352 z+3.6624}
\end{array}\right] .
$$


Applying the long division operation yields

$$
(z I-A)^{-1} z=\left[\begin{array}{cc}
1+\frac{2.856 z-3.6624}{z^{2}-3.8352 z+3.6624} & \frac{0.0408 z}{z^{2}-3.8352 z+3.6624} \\
\frac{0.0816 z}{z^{2}-3.8352 z+3.6624} & 1+\frac{2.8965 z-3.6624}{z^{2}-3.8352 z+3.6624}
\end{array}\right] .
$$

Then, use the general partial fraction expansion to get

$$
(z I-A)^{-1} z=\left[\begin{array}{cc}
1+\frac{8.8877}{z-2.0392}+\frac{-6.0321}{z-1.796} & \frac{0.3421}{z-2.0392}+\frac{-0.3014}{z-1.796} \\
\frac{0.6842}{z-2.0392}+\frac{-0.6028}{z-1.796} & 1+\frac{9.2299}{z-2.0392}+\frac{-6.3331}{z-1.796}
\end{array}\right] .
$$

Using inverse $z$ transformation yields the solution to be where

$$
X(k)=Z^{-1}\left[(z I-A)^{-1} z\right] X(0) \equiv\left[\begin{array}{ll}
X_{11} & X_{12} \\
X_{21} & X_{22}
\end{array}\right]\left[\begin{array}{c}
2 \times 10^{7} \\
18 \times 10^{7}
\end{array}\right]
$$

$$
\begin{aligned}
& X_{11} \equiv \delta(k)+(8.8877)(2.0392)^{k-1} u_{s}(k-1)+(-6.0321)(1.796)^{k-1} u_{s}(k-1), \\
& X_{12} \equiv(0.3421)(2.0392)^{k-1} u_{s}(k-1)-(0.3014)(1.796)^{k-1} u_{s}(k-1), \\
& X_{21} \equiv(0.6842)(2.0392)^{k-1} u_{s}(k-1)-(0.6028)(1.796)^{k-1} u_{s}(k-1), \\
& X_{22} \equiv \delta(k)+(0.6842)(2.0392)^{k-1} u_{s}(k-1)-(6.3331)(1.796)^{k-1} u_{s}(k-1) .
\end{aligned}
$$

To compare the proposed method with traditional approach, we use Theorem 1 to solve same problem without using the above polynomial multiplication and long division. Directly do partial fraction expansion to equation (101), and we can easily get

$$
(z I-A)^{-1} z=\left[\begin{array}{cc}
1+\frac{A}{z-2.0392}+\frac{B}{z-1.796} & \frac{C}{z-2.0392}+\frac{D}{z-1.796} \\
\frac{E}{z-2.0392}+\frac{F}{z-1.796} & 1+\frac{G}{z-2.0392}+\frac{H}{z-1.796}
\end{array}\right]
$$


where $A=8.8877, B=-6.0321, C=0.3421, D=-0.3014$, where $E=0.6842, F=-0.6028, G=9.2299, H=-6.3331$. Using Theorem 1 yields the solution to be

$$
X(k)=\mathrm{Z}^{-1}\left[(z I-A)^{-1} z\right] X(0) \equiv\left[\begin{array}{ll}
X_{11} & X_{12} \\
X_{21} & X_{22}
\end{array}\right]\left[\begin{array}{c}
2 \times 10^{7} \\
18 \times 10^{7}
\end{array}\right]
$$

$$
\begin{aligned}
& X_{11} \equiv \delta(k)+(8.8877)(2.0392)^{k-1} u_{s}(k-1)+(-6.0321)(1.796)^{k-1} u_{s}(k-1), \\
& X_{12} \equiv(0.3421)(2.0392)^{k-1} u_{s}(k-1)-(0.3014)(1.796)^{k-1} u_{s}(k-1), \\
& X_{21} \equiv(0.6842)(2.0392)^{k-1} u_{s}(k-1)-(0.6028)(1.796)^{k-1} u_{s}(k-1), \\
& X_{22} \equiv \delta(k)+(0.6842)(2.0392)^{k-1} u_{s}(k-1)-(6.3331)(1.796)^{k-1} u_{s}(k-1) .
\end{aligned}
$$

Then, we can conclude that the predicted population of the kth year for B city is

$$
\begin{aligned}
x_{1}(k+1) \equiv & \left(2 \times 10^{7}\right)\left\{\delta(k)+(8.8877)(2.0392)^{k-1} u_{s}(k-1)+(-6.0321)(1.796)^{k-1} u_{s}(k-1)\right\} \\
& +\left(18 \times 10^{7}\right)\left\{(0.3421)(2.0392)^{k-1} u_{s}(k-1)-(0.3014)(1.796)^{k-1} u_{s}(k-1)\right\},
\end{aligned}
$$

and the predicted population of the country other than the B city is

$$
\begin{aligned}
x_{2}(k+1) \equiv & \left(2 \times 10^{7}\right)\left\{(0.6842)(2.0392)^{k-1} u_{s}(k-1)-(0.6028)(1.796)^{k-1} u_{s}(k-1)\right\} \\
& +\left(18 \times 10^{7}\right)\left\{\delta(k)+(0.6842)(2.0392)^{k-1} u_{s}(k-1)-(6.3331)(1.796)^{k-1} u_{s}(k-1)\right\}
\end{aligned}
$$

4.2. [Application 2]. Market Price Balance Analysis. The watermelon supply function is known as

$$
y(k+1)=-0.5+8(p(k)) \equiv-c+d(p(k)),
$$

and the watermelon demand function is

$$
x(k+1)=7-12(p(k+1)) \equiv a-b(p(k+1)),
$$

where the market balance price is defined as $\mathrm{p}(k)$. Next, the market price stability analysis will be carried out, and then the market balance price will be obtained. When the market reaches the balance between supply and demand, the following equation can be derived.

$$
a-b(p(k+1))=-c+d(p(k)),
$$

that is,

$$
p(k+1)=\left(-\frac{d}{b}\right) p(k)+\left(\frac{a+c}{b}\right) .
$$

Taking the z-transformation of the above equation yields a rational fraction $p(z)$ with distinct poles, where the numerator and the denominator are in the same power

$$
\begin{aligned}
P(z) & =\left(\frac{z}{z+(d / b)}\right) p(0)+\left(\frac{a+c}{b}\right)\left[\frac{z}{(z+(d / b))(z-1)}\right] \\
& =\frac{p(0) z^{2}+[-p(0)+(a+c / b)] z}{(z+(d / b))(z-1)} .
\end{aligned}
$$

For the traditional method [20,21], the denominator and the numerator polynomial must be multiplied first to facilitate the long division operation because the denominator and the numerator have the same order.

$$
\begin{aligned}
P(z) & =\left(\frac{z}{z+(d / b)}\right) p(0)+\left(\frac{a+c}{b}\right)\left[\frac{z}{(z+(d / b))(z-1)}\right] \\
& =\frac{p(0) z^{2}+[-p(0)+(a+c / b)] z}{z^{2}+(d / b-1) z-d / b} .
\end{aligned}
$$


Using the long division operation gets

$$
P(z)=p(0)+\frac{[a+c / b-p(0) d / b] z+p(0) d / b}{z^{2}+(d / b-1) z-d / b} .
$$

Then, apply the general partial fraction expansion to obtain

$$
\begin{aligned}
P(z)= & p(0)+\frac{(-d / b) p(0)+(a+c) d /(b+d) b}{z+(d / b)} \\
& +\frac{(a+c / b+d)}{z-1} .
\end{aligned}
$$

Applying inverse $z$-transformation gets the solution

$$
p(k)=p(0) \delta(k)+\left[\left(-\frac{d}{b}\right) p(0)+\frac{(a+c) d}{(b+d) b}\right]\left(-\frac{d}{b}\right)^{k-1} u_{s}(k-1)+\left(\frac{a+c}{b+d}\right)(1)^{k-1} u_{s}(k-1)
$$

To compare the proposed method with traditional approach, we apply Theorem 1 to address same problem without using the above polynomial multiplication and long division. Directly apply partial fraction expansion to equation (116), and we can easily get

$$
\begin{aligned}
P(z)= & p(0)+\frac{(-d / b) p(0)+(a+c) d /(b+d) b}{z+(d / b)} \\
& +\frac{(a+c / b+d)}{z-1} .
\end{aligned}
$$

$$
p(k)=p(0) \delta(k)+\left[\left(-\frac{d}{b}\right) p(0)+\frac{(a+c) d}{(b+d) b}\right]\left(-\frac{d}{b}\right)^{k-1} u_{s}(k-1)+\left(\frac{a+c}{b+d}\right)(1)^{k-1} u_{s}(k-1)
$$

It can be seen from the above formula that when $d / b$ is less than 1 , we can obtain $\lim _{k \rightarrow \infty}(d / b)^{k}=0$. Therefore, the solution of the market price model tends to a fixed constant

$$
\lim _{k \rightarrow \infty} p(k)=\frac{a+c}{b+d}
$$

which concludes that the market price is stable, and this fixed constant is then the market balance price when the supply and demand are balanced.

\section{3. [Application 3]. Analysis of Loan Economic Activities.} Someone loans 15,000 dollars for the project to open, where the monthly interest rate of the loan is $1 \%$, and the monthly repayment is 200 dollars. Assume that the loan balance at the end of the $k$ th month is $x(k)$, the repayment amount is $p(k)$, and the monthly interest rate is $i$. The solution of the loan balance $x(k)$ at the end of the $k$ th month is as follows. According to the above statements, the system state equation for this economic activity is

$$
x(k+1)=(1+i) x(k)-p(k),
$$

where $x(0)=15000, i=0.01, p(k)=200$.

That is,

$$
x(k+1)=1.01 x(k)-200 .
$$

According to Theorem 1, the following equation can be obtained.
Taking the $z$-transformation of the above equation yields $X(z)$ with distinct poles, where the numerator and the denominator are in the same power

$$
X(z)=\frac{15000 z}{z-1.01}-\frac{200 z}{(z-1.01)(z-1)}=\frac{15000 z^{2}-15200 z}{(z-1.01)(z-1)} .
$$

Since the order of the denominator and the numerator is the same, the denominator and the numerator polynomial must be multiplied first to facilitate the long division operation when we use the traditional method $[20,21]$,

$$
X(z)=\frac{15000 z^{2}-15200 z}{z^{2}-2.01 z+1.01} .
$$

Utilize the long division operation to derive

$$
X(z)=15000+\frac{14950 z-15150}{z^{2}-2.01 z+1.01} .
$$

Using the general partial fraction expansion gets

$$
X(z)=15000+\frac{-5050}{z-1.01}+\frac{20000}{z-1} .
$$

Then, using inverse $z$-transformation obtains 


$$
\begin{aligned}
x(k)= & 15000 \delta(k)+(-5050)(1.01)^{k-1} u_{s}(k-1) \\
& +20000(1)^{k-1} u_{s}(k-1) .
\end{aligned}
$$

To compare the proposed method with traditional approach, we use Theorem 1 to solve the same problem without using the above polynomial multiplication and long division. Directly apply partial fraction expansion to equation (126), and we can easily get

$$
X(z)=15000+\frac{-5050}{z-1.01}+\frac{20000}{z-1} .
$$

Consequently, according to Theorem 1, we can conclude that the solution of the loan balance $x(k)$ at the end of the $k$ th month is

$$
\begin{aligned}
x(k)= & 15000 \delta(k)+(-5050)(1.01)^{k-1} u_{s}(k-1) \\
& +20000(1)^{k-1} u_{s}(k-1) .
\end{aligned}
$$

4.4. [Application 4]. Real Estate Market Price System. Assuming that the real estate supply, demand, and price data of a country from 2000 to 2009 are known as shown in Table 1, this example is to study the stability of the real estate market price system [28]. First use the linear regression analysis to obtain the following demand function and supply function:

$$
\begin{aligned}
D_{\text {em }}(k) & =-573.4106+0.3563 P_{\text {re }}(k), \\
S_{\text {up }}(k) & =-687.0668+0.4306 P_{\text {re }}(k-1) .
\end{aligned}
$$

Compare the demand function and the supply function to get $0.4306>0.3563$. So, this real estate market price system is divergent and unstable. For such a system, it will bring more and more oscillations with the increase of time and result in instability of the supply and demand system. If it is not controlled, it will make the real estate market price supply and demand system out of balance. Next, construct a mathematical state-space model of the unbalanced real estate market price containing the expected price.

$$
\begin{aligned}
& D_{\mathrm{em}}(k)=\alpha-\beta P_{\mathrm{re}}(k), \\
& S_{\mathrm{up}}(k)=-\delta+\Gamma P_{\mathrm{re}}^{*}(k), \\
& P_{\mathrm{re}}^{*}(k)=P_{\mathrm{re}}(k-1)+\gamma\left[D_{\mathrm{em}}(k-1)-S_{\mathrm{up}}(k-1)\right],
\end{aligned}
$$

where $P_{\text {re }}^{*}(k)$ represents the producer's expected price for the $k$ th real estate. From equations (134) (136), we can get the difference equation as follows:

$$
\begin{aligned}
D_{\mathrm{em}}(k+1)-S_{\mathrm{up}}(k+1)= & \alpha-\beta P_{\mathrm{re}}(k+1)+\delta-\Gamma\left\{P_{\mathrm{re}}(k)+\gamma\left[D_{\mathrm{em}}(k)-S_{\mathrm{up}}(k)\right]\right\} \\
= & \alpha-\beta P_{\mathrm{re}}(k+1)+\delta-\Gamma\left\{P_{\mathrm{re}}(k)+\gamma\left[D_{\mathrm{em}}(k)-S_{\mathrm{up}}(k)\right]\right\}+\left[D_{\mathrm{em}}(k)-S_{\text {up }}(k)\right] \\
& -\left[D_{\mathrm{em}}(k)-S_{\mathrm{up}}(k)\right],
\end{aligned}
$$

that is,

$$
\begin{aligned}
D_{\text {em }}(k+1)-S_{\text {up }}(k+1)= & (1-\Gamma \gamma)\left[D_{\text {em }}(k)-S_{\text {up }}(k)\right]-\beta\left[P_{\text {re }}(k+1)-P_{\text {re }}(k)\right] \\
& +\Gamma \gamma\left[D_{\text {em }}(k-1)-S_{\text {up }}(k-1)\right]-\Gamma\left[P_{\text {re }}(k)-P_{\text {re }}(k-1)\right] .
\end{aligned}
$$

Define the state-space variables and input to be

$$
\begin{aligned}
& x_{1}(k) \equiv D_{\mathrm{em}}(k)-S_{\mathrm{up}}(k), \\
& x_{2}(k) \equiv D_{\mathrm{em}}(k-1)-S_{\mathrm{up}}(k-1), \\
& x_{3}(k) \equiv P_{\mathrm{re}}(k)-P_{\mathrm{re}}(k-1) \equiv u(k) .
\end{aligned}
$$

And then the state equation and output equation are given by

$$
\begin{gathered}
{\left[\begin{array}{c}
x_{1}(k+1) \\
x_{2}(k+1) \\
x_{3}(k+1)
\end{array}\right]=\left[\begin{array}{rrr}
(1-\Gamma \gamma) & \Gamma \gamma & -\gamma \\
1 & 0 & 0 \\
0 & 0 & 0
\end{array}\right]\left[\begin{array}{l}
x_{1}(k) \\
x_{2}(k) \\
x_{3}(k)
\end{array}\right]+\left[\begin{array}{c}
-\beta \\
0 \\
1
\end{array}\right] u(k),} \\
y(k)=\left[\begin{array}{lll}
1 & 0 & 0
\end{array}\right]\left[\begin{array}{l}
x_{1}(k) \\
x_{2}(k) \\
x_{3}(k)
\end{array}\right]=x_{1}(k) .
\end{gathered}
$$


Considering the impact of the magnitude and speed of the price adjustment on the allocation of resources, the new dynamic equation can be obtained by taking the price adjustment parameter as $\gamma=0.1, \Gamma=0.4306, \beta=0.3563$ :

$$
\begin{gathered}
{\left[\begin{array}{l}
x_{1}(k+1) \\
x_{2}(k+1) \\
x_{3}(k+1)
\end{array}\right]=\left[\begin{array}{ccc}
0.95694 & 0.04306 & -0.04306 \\
1 & 0 & 0 \\
0 & 0 & 0
\end{array}\right]\left[\begin{array}{l}
x_{1}(k) \\
x_{2}(k) \\
x_{3}(k)
\end{array}\right]+\left[\begin{array}{c}
-0.3563 \\
0 \\
1
\end{array}\right] u(k),} \\
y(k)=\left[\begin{array}{lll}
1 & 0 & 0
\end{array}\right]\left[\begin{array}{l}
x_{1}(k) \\
x_{2}(k) \\
x_{3}(k)
\end{array}\right] \equiv C\left[\begin{array}{l}
x_{1}(k) \\
x_{2}(k) \\
x_{3}(k)
\end{array}\right]=x_{1}(k) \equiv A\left[\begin{array}{l}
x_{1}(k) \\
x_{2}(k) \\
x_{3}(k)
\end{array}\right]+B u(k) .
\end{gathered}
$$
follows:

Apply the z-transformation to solve the solution as

$$
(z I-A)^{-1} z=\left[\begin{array}{ccc}
\frac{z^{2}}{(z-0.9999)(z+0.0430)} & \frac{0.0431 z}{(z-0.9999)(z+0.0430)} & \frac{-0.4306 z}{(z-0.9999)(z+0.0430)} \\
\frac{z}{(z-0.9999)(z+0.0430)} & \frac{z^{2}-0.9569 z}{(z-0.9999)(z+0.0430)} & \frac{-0.4306}{(z-0.9999)(z+0.0430)} \\
0 & 0 & \frac{1}{z}
\end{array}\right] .
$$

We first use the traditional method to solve it. For the traditional method, the denominator and the numerator polynomial must be multiplied first to facilitate the long division operation because the denominator and the numerator have the same order.

$$
(z I-A)^{-1} z=\left[\begin{array}{ccc}
\frac{z^{2}}{z^{2}-0.9569 z-0.0430} & \frac{0.0431 z}{z^{2}-0.9569 z-0.0430} & \frac{-0.4306 z}{z^{2}-0.9569 z-0.0430} \\
\frac{z}{z^{2}-0.9569 z-0.0430} & \frac{z^{2}-0.9569 z}{z^{2}-0.9569 z-0.0430} & \frac{-0.4306}{z^{2}-0.9569 z-0.0430} \\
0 & 0 & \frac{1}{z}
\end{array}\right]
$$

Using the long division operation gets

$$
(z I-A)^{-1} z=\left[\begin{array}{cccc}
1+\frac{0.9569 z+0.0430}{z^{2}-0.9569 z-0.0430} & \frac{0.0431 z}{z^{2}-0.9569 z-0.0430} & \frac{-0.4306 z}{z^{2}-0.9569 z-0.0430} \\
\frac{z}{z^{2}-0.9569 z-0.0430} & 1+\frac{0.0430}{z^{2}-0.9569 z-0.0430} & \frac{-0.4306}{z^{2}-0.9569 z-0.0430} \\
0 & 0 & \frac{1}{z}
\end{array}\right] .
$$


Then, apply the partial fraction expansion to obtain

$$
(z I-A)^{-1} z=\left[\begin{array}{ccc}
1+\frac{0.9587}{z-0.9999}+\frac{-0.0018}{z+0.0430} & \frac{0.0412}{z-0.9999}+\frac{0.0018}{z+0.0430} & \frac{-0.4128}{z-0.9999}+\frac{-0.0178}{z+0.0430} \\
\frac{0.9588}{z-0.9999}+\frac{0.0412}{z+0.0430} & 1+\frac{0.0412}{z-0.9999}+\frac{-0.0412}{z+0.0430} & \frac{-0.4129}{z-0.9999}+\frac{0.4129}{z+0.0430} \\
0 & 0 & \frac{1}{z}
\end{array}\right]
$$

Using inverse $z$-transformation yields the solution to be

$$
X(k) \equiv\left[\begin{array}{l}
x_{1}(k) \\
x_{2}(k) \\
x_{3}(k)
\end{array}\right]=Z^{-1}\left[(z I-A)^{-1} z\right] X(0) \equiv\left[\begin{array}{lll}
X_{11} & X_{12} & X_{13} \\
X_{21} & X_{22} & X_{23} \\
X_{31} & X_{32} & X_{33}
\end{array}\right]\left[\begin{array}{l}
x_{1}(0) \\
x_{2}(0) \\
x_{3}(0)
\end{array}\right]
$$

where

$$
\begin{aligned}
& X_{11} \equiv \delta(k)+(0.9587)(0.9999)^{k-1} u_{s}(k-1)+(-0.0018)(-0.0430)^{k-1} u_{s}(k-1), \\
& X_{12} \equiv(0.0412)(0.9999)^{k-1} u_{s}(k-1)+(0.0018)(-0.0430)^{k-1} u_{s}(k-1), \\
& X_{13} \equiv(-0.4128)(0.9999)^{k-1} u_{s}(k-1)+(-0.0178)(-0.0430)^{k-1} u_{s}(k-1), \\
& X_{21} \equiv(0.9588)(0.9999)^{k-1} u_{s}(k-1)+(0.0412)(-0.0430)^{k-1} u_{s}(k-1), \\
& X_{22} \equiv \delta(k)+(0.0412)(0.9999)^{k-1} u_{s}(k-1)+(-0.0412)(-0.0430)^{k-1} u_{s}(k-1), \\
& X_{23} \equiv(-0.4129)(0.9999)^{k-1} u_{s}(k-1)+(0.4129)(-0.0430)^{k-1} u_{s}(k-1), \\
& X_{31} \equiv 0, \\
& X_{32} \equiv 0, \\
& X_{33} \equiv \delta(k-1) .
\end{aligned}
$$

Then, we can get the state variables to be

$$
\begin{aligned}
& x_{1}(k)=X_{11} x_{1}(0)+X_{12} x_{2}(0)+X_{13} x_{3}(0), \\
& x_{2}(k)=X_{21} x_{1}(0)+X_{22} x_{2}(0)+X_{23} x_{3}(0), \\
& x_{3}(k)=X_{31} x_{1}(0)+X_{32} x_{2}(0)+X_{33} x_{3}(0) .
\end{aligned}
$$

To compare the proposed method with traditional approach, we use Theorem 1 to solve the same problem without using the above polynomial multiplication and long division. Directly do partial fraction expansion to equation (142), and we can easily get 
TABle 1: The supply, demand, and price of real estate in a country from 2000 to 2009.

\begin{tabular}{lccc}
\hline Year & Supply $/ 10^{\wedge} 5 \mathrm{~m}^{\wedge} 2$ & Demand $/ 10^{\wedge} 5 \mathrm{~m}^{\wedge} 2$ & Price $/ \mathrm{m}^{\wedge} 2$ \\
\hline 2000 & 44985.5 & 9010.17 & 1997 \\
2001 & 50770.1 & 12185.30 & 2063 \\
2002 & 56857.6 & 14556.53 & 2053 \\
2003 & 65896.9 & 18637.13 & 2112 \\
2004 & 79411.7 & 22411.90 & 2170 \\
2005 & 94104.0 & 26808.29 & 2250 \\
2006 & 117526.0 & 33717.63 & 2359 \\
2007 & 140451.4 & 38231.64 & 2778 \\
2008 & 166053.3 & 55486.22 & 3168 \\
2009 & 194786.4 & 61857.07 & 3367 \\
\hline
\end{tabular}

$$
(z I-A)^{-1} z=\left[\begin{array}{ccc}
1+\frac{0.9587}{z-0.9999}+\frac{-0.0018}{z+0.0430} & \frac{0.0412}{z-0.9999}+\frac{0.0018}{z+0.0430} & \frac{-0.4128}{z-0.9999}+\frac{-0.0178}{z+0.0430} \\
\frac{0.9588}{z-0.9999}+\frac{0.0412}{z+0.0430} & 1+\frac{0.0412}{z-0.9999}+\frac{-0.0412}{z+0.0430} & \frac{-0.4129}{z-0.9999}+\frac{0.4129}{z+0.0430} \\
0 & 0 & \frac{1}{z}
\end{array}\right]
$$

Equation (149) is the same as equation (145) that is derived with traditional method. From equation (149), the statespace model of the unbalanced real estate market price containing the expected price is convergent and stable. In summary, it can be concluded that the proposed method is simpler and more effective than traditional methods without using the above polynomial multiplication and long division.

\section{Conclusion}

We propose a direct method used to solve the linear nonhomogeneous time-invariant difference equation with the same number for inputs and outputs in this study and explicitly express the general form of the solution sequence with shorter time than that of the traditional approaches. Overall, the proposed direct approach in this study has more generality and universality with more efficiency and provides a significant solving method for the rational $z$ function with the same power of the numerator and the denominator. As we know, the traditional methods used for solving linear nonhomogeneous difference equations face several difficulties in practical applications. The main contributions of the proposed approach are to find the solution without inevitable troublesome operations including the multiplication of all polynomial factors, the long division of two complex polynomials, the differentiation of two complex polynomials, and the derivations of the complex partial fraction expansion variables for the existing traditional approaches. Some practical applications of solving the recurrence difference equation including the economic cybernetics in population prediction, analysis of loan economic activities, and market price balance analysis are investigated in this study.

\section{Data Availability}

No data were used to support this study.

\section{Conflicts of Interest}

The authors declare that they have no conflicts of interest.

\section{Acknowledgments}

The financial supports from the Office of Philosophy and Social Science Research Project of Guang Dong Province, China (GD17XYJ29) and the City College of Dongguan University of Technology (2017YZDYB01Z) are gratefully acknowledged.

\section{References}

[1] R. P. Agarwal, Difference Equations and Inequalities: Theory, Methods, and Applications, CRC Press, Boca Raton, FL, USA, 2000.

[2] C. T. Chen, Linear System Theory and Design, Oxford University Press, New York, NY, USA, 1999.

[3] L. Ljung, System Identification - Theory for the User, PrenticeHall, Upper Saddle River, NJ, USA, 1999.

[4] O. Yoshifumi, Discrete Control Systems, Springer, London, UK, 2013.

[5] A. V. Oppenheim, R. W. Schafer, and J. R. Buck, Discrete-Time Signal Processing, Prentice-Hall, Upper Saddle River, NJ, USA, 1999.

[6] R. A. Brualdi, Introductory Combinatorics, Prentice-Hall, Upper Saddle River, NJ, USA, 2009.

[7] S. L. Sun and Y. L. Xu, Introductory Combinatorics, USTC Press, Hefei, China, 1999.

[8] L. Jiang, J. A. Fang, and W. N. Zhou, "Stability analysis of economic discrete-time singular dynamic input-output 
model," in Proceedings of the 2008 International Conference on Machine Learning and Cybernetics, vol. 3, pp. 1434-1438, Kunming, Chinavol, July 2008.

[9] A. L. Klyuenkov, "Regulation of macroeconomic trends based on the dynamical difference input-output model," in Proceedings of the 2015 International Conference Stability and Control Processes, pp. 467-469, Saint Petersburg, Russia, October 2015.

[10] P. Stakhiv, F. Yu, and S. Moskalyk, "Mathematical modeling of complex dynamic technical and economic systems," Modern Problems of Radio Engineering, Telecommunications and Computer Science, pp. 106-107, 2002.

[11] B. Bao, J. Ma, and M. Goh, "Short- and long-term repeated game behaviours of two parallel supply chains based on government subsidy in the vehicle market," International Journal of Production Research, vol. 57, p. 25, 2019.

[12] L. Xie, J. Ma, and M. Goh, "Supply chain coordination in the presence of uncertain yield and demand," International Journal of Production Research, vol. 58, p. 18, 2020.

[13] J. Ma, Y. Hou, W. Yang, and Y. Tian, "A time-based pricing game in a competitive vehicle market regarding the intervention of carbon emission reduction," Energy Policy, vol. 142, pp. 1-12, Article ID 111440, 2020.

[14] A. M. Elaiw, A. A. Raezah, and S. A. Azoz, "Stability of delayed HIV dynamics models with two latent reservoirs and immune impairment," Advances in Difference Equations, vol. 2018, no. 414, pp. 1-25, 2018.

[15] R. P. Grimaldi, Discrete and Combinatorial Mathematics, Addison-Wesley, New York, NY, USA, 1989.

[16] Y. Shen and Y. Li, "The z-transform method for the ulam stability of linear difference equations with constant coefficients," Advances in Difference Equations, vol. 2018, no. 396, pp. 1-15, 2018.

[17] A. M. Davis, "A simple way of obtaining the reduced Jordan form of a state equation," IEEE Transactions on Education, vol. 47, no. 4, pp. 481-489, 2004.

[18] C. T. Chen, Digital Signal Processing, Oxford University Press, New York, NY, USA, 2001.

[19] G. F. Franklin, J. D. Powell, and M. Workman, Digital Control of Dynamic Systems, Addison Wesley Longman, California, UK, 1998.

[20] S. K. Mitra, Digital Signal Processing, McGraw-Hill, New York, NY, USA, 2001.

[21] K. Ogata, Discrete-time Control Systems, Prentice-Hall, New Jersey, NJ, USA, 1995.

[22] A. V. Oppenheim and R. W. Schafer, Digital Signal Processing, Prentice-Hall, Englewood Cliffs, Bergen, 1975.

[23] A. V. Oppenheim, A. S. Willsky, and S. H. Nawab, Signals and Systems, Prentice-Hall, New Jersey, NY, USA, 1997.

[24] R. E. Ziemer, W. H. Tranter, and D. R. Fannin, Signals and Systems, Prentice-Hall, New Jersey, NJ, USA, 1998.

[25] R. V. Churchill and J. W. Brown, Introduction to Complex Variables and Applications, McGraw-Hill, New York, NY, USA, 1990.

[26] B. C. Kuo, Discrete Control Systems, Prentice-Hall, New Jersey, NJ, USA, 1970.

[27] C. L. Phillips and H. T. Nagle, Digital Control System Analysis and Design, Prentice-Hall, New Jersey, NJ, USA, 1995.

[28] J. Wang, Z. Wang, N. Lu, and Z. Zhen, Economic Cybernetics, Elsevier, Beijing, China, 2008. 